\title{
HOCHSCHILD-PIRASHVILI HOMOLOGY ON SUSPENSIONS AND REPRESENTATIONS OF $\operatorname{Out}\left(F_{n}\right)$
}

\author{
VICTOR TURCHIN AND THOMAS WILLWACHER
}

\begin{abstract}
Aвstract. We show that the Hochschild-Pirashvili homology on any suspension admits the so called Hodge splitting. For a map between suspensions $f: \Sigma Y \rightarrow \Sigma Z$, the induced map in the Hochschild-Pirashvili homology preserves this splitting if $f$ is a suspension. If $f$ is not a suspension, we show that the splitting is preserved only as a filtration. As a special case, we obtain that the Hochschild-Pirashvili homology on wedges of circles produces new representations of $\operatorname{Out}\left(F_{n}\right)$ that do not factor in general through $\operatorname{GL}(n, \mathrm{Z})$. The obtained representations are naturally filtered in such a way that the action on the graded quotients does factor through $\operatorname{GL}(n, \mathrm{Z})$.
\end{abstract}

\section{INTRODUCTION}

The higher Hochschild homology is a bifunctor introduced by T. Pirashvili in [28] that to a topological space (simplicial set) and a (co)commutative (co)algebra assigns a graded vector space. Informally speaking this functor is a way to "integrate" a (co)algebra over a given space. Specialized to a circle the result is the usual Hochschild homology. The precursor to the higher Hochschild homology was the discovery of the Hodge splitting in the usual Hochschild homology of a commutative algebra [13, 21]. Indeed, the most surprising and perhaps the motivating result for T. Pirashvili to write his seminal work [28] was the striking fact that the higher Hochschild homology on a sphere of any positive dimension also admits the Hodge splitting and moreover the terms of the splitting up to a regrading depend only on the parity of the dimension of the sphere. With this excuse to be born, the higher Hochschild homology is nowadays a widely used tool that has various applications including the string topology and more generally the study of mapping and embedding spaces [28, 1, 2, 15, 25, 26, 30, 31]. It also has very interesting and deep generalizations such as the topological higher Hochschild homology [8, 29] and factorization homology [3, 14, 16, 23].

In our work we study the very nature of the Hodge splitting. In particular we show that it always takes place for suspensions. Moreover, it will be clear from the construction that only suspensions and spaces rationally homology equivalent to them have this property. For any suspension $\Sigma Y$, the terms of the splitting depend in some polynomial way on $\tilde{H}_{*} \Sigma Y$, which in particular explains Pirashvili's result for spheres. We also show that if a map $f: \Sigma Y \rightarrow \Sigma Z$ is a suspension, than the induced map in the Hochschild-Pirashvili homology preserves the splitting and is determined by the map $f_{*}: \tilde{H}_{*} \Sigma Y \rightarrow \tilde{H}_{*} \Sigma Z$. In case $f$ is not a suspension, the Hodge splitting is preserved only as a filtration. We explain how the induced map between different layers is computed from the rational homotopy type of $f$.

We treat more carefully the case of wedges of circles and discover certain representations of the group $\operatorname{Out}\left(F_{n}\right)$ of outer automorphisms of a free groun $]^{1}$ that have the smallest known dimension among those that don't factor through $\mathrm{GL}(n, \mathbb{Z})$.

Notation. We work over rational numbers $\mathbb{Q}$ unless otherwise stated. All vector spaces are assumed to be vector spaces over $\mathbb{Q}$. Graded vector spaces are vector spaces with a $\mathbb{Z}$-grading, and we abbreviate the phrase "differential graded" by dg as usual. We generally use homological conventions, i.e., the differentials will have degree -1 . We denote by $g V e c t$ and $d g V e c t$ the category of graded vector spaces and the category of chain complexes respectively. For a chain complex or a graded vector space $C$ we denote by $C[k]$ its $k$-th desuspension.

We use freely the language of operads. A good introduction into the subject can be found in the textbook [22], whose conventions we mostly follow. We use the notation $\mathcal{P}\{k\}$ for the $k$-fold operadic suspension. The operads governing commutative, associative and Lie algebras are denoted by Com, Assoc, and Lie respectively. By Com we denote the commutative non-unital operad and by coLie the cooperad dual to Lie.

For a category $C$, we denote by mod-C the category of cofunctors $C^{o p} \rightarrow d g V e c t$ to chain complexes. The objects of mod- $C$ will be called right $C$-modules. In the following section, $C$ is either the category $\Gamma$ of finite

2010 Mathematics Subject Classification. 55N99;19D55;13D03.

V.T. acknowledges partial support by the MPIM, Bonn, and the IHES . T.W. acknowledges partial support by the Swiss National Science Foundation (grant 200021_150012) and the SwissMap NCCR, funded by the Swiss National Science Foundation.

${ }^{1}$ These representations appear as application to the hairy graph-homology computations in the study of the spaces of long embeddings, higher dimensional string links, and the deformation theory of the little discs operads [2] 31, 32 33, 34]. 
pointed sets or the category Fin of finite sets. Abusing notation we denote the set $\{1, \ldots, k\}$ by $k$ and the set $\{*, 1, \ldots, k\}$ based at $*$ by $k_{*}$. We will consider the following examples of right $\Gamma$ and Fin-modules:

- For $X$ some topological space we can consider the Fin-module sending a finite set $S$ to the singular chains on the mapping space $C_{*}\left(X^{S}\right)$. We denote this Fin-module by $C_{*}\left(X^{\bullet}\right)$.

- Similarly, to a basepointed space $X_{*}$ we assign a $\Gamma$-module $C_{*}\left(X_{*}^{\bullet}\right)$ sending a pointed set $S_{*}$ to $C_{*}\left(X_{*}^{S_{*}}\right)$, where now $X_{*}^{S_{*}}$ is supposed to be the space of pointed maps.

- To a cocommutative coalgebra $C$ we assign the Fin-module sending the finite set $S$ to the tensor product $C^{\otimes S} \cong \bigotimes_{s \in S} C$. We denote this Fin-module by $C^{\otimes \bullet}$. If not otherwise stated we assume that $C$ is nonnegatively graded and simply connected.

- If in addition $M$ is a $C$-comodule (e.g., $M=C$ ) one can construct a $\Gamma$-module $M \otimes C^{\otimes \bullet}$ such that $S_{*} \mapsto$ $M \otimes \bigotimes_{s \in S_{*} \backslash\{*\}} C$.

- Dually, if $M$ is a module over a commutative algebra $A$, then $M \otimes A^{\otimes \bullet}$ is a left $\Gamma$-module, and its objectwise dual $\left(M \otimes A^{\otimes \bullet}\right)^{\vee}$ is a right $\Gamma$-module.

A topological space is said of finite type if all its homology groups are finitely generated in every degree.

Two spaces are said rationaly homology equivalent if there is a zigzag of maps between them, such that its every map induces an isomorphism in rational homology.

The completed tensor product is denoted by $\hat{\otimes}$.

Main results. In the paper for simplicity of exposition we stick to the contravariant Hochschild-Pirashvili homology that is to the one assigned to right Fin and $\Gamma$ modules. One should mention however that all the results can be easily adjusted to the covariant case as well.

There are two ways to define the higher Hochschild homology. In the first combinatorial way, for a space $X$ (respectively pointed space $X_{*}$ ) obtained as a realization of a (pointed) finite simplicial set $X_{\bullet}: \Delta^{o p} \rightarrow$ Fin (respectively $\mathcal{X}_{\bullet}: \Delta^{o p} \rightarrow \Gamma$ ), the higher Hochschild homology $H H^{X}(L)$ (respectively $H H^{X_{*}}\left(L_{*}\right)$ ) can be computed as the homology of the totalization of the cosimplicial chain complex $L \circ \mathcal{X}: \Delta \rightarrow \operatorname{dgVect}$ (respectively $L_{*} \circ$ $\mathcal{X}_{*}: \Delta \rightarrow d g$ Vect). 2

In another definition, for a right Fin-module $L$ (respectively right $\Gamma$-module $L_{*}$ ) and a topological space $X$ (respectively pointed space $X_{*}$ ), the higher Hochschild homology that we also call Hochschild-Pirashvili homology $H H^{X}(L)$ (respectively $H H^{X_{*}}\left(L_{*}\right)$ ) is the homology of the complex of homotopy natural transformations $C_{*}\left(X^{\bullet}\right) \rightarrow$ $L\left(\right.$ respectively $\left.C_{*}\left(X_{*}^{*}\right) \rightarrow L_{*}\right)[28,16$

The fact that the two definitions are equivalent is implicitly shown in the proof of [28, Theorem 2.4] by Pirashvili, see also [16, Proof of Proposition 4] and [30, Proposition 3.4].

In case $L=C^{\otimes \bullet}$ (respectively $L=M \otimes C^{\otimes \bullet}$ ), we denote the higher Hochschild homology as $H H^{X}(C)$ (respectively $\left.H^{X_{*}}(C, M)\right)$ 尚

In our paper the combinatorial definition will be used only for wedges of circles as we want to treat this case more explicitly. Later in the paper we show that for wedges of circles the first and the second definitions produce identical complexes.

Any map $f: X \rightarrow Y$ (respectively basepoint preserving map $X_{*} \rightarrow Y_{*}$ ) induces a map $f^{*}: H H^{Y}(L) \rightarrow H H^{X}(L)$ (respectively $H H^{Y_{*}}\left(L_{*}\right) \rightarrow H H^{X_{*}}\left(L_{*}\right)$ ). Two homotopic maps (respectively basepoint homotopic maps) induce the same map in higher Hochschild homology. It is also clear from the (first) definition that in case $f$ is a rational homology equivalence, then the induced map $f^{*}$ is an isomorphism. One has a functor $u: \Gamma \rightarrow$ Fin that forgets the basepoint. If $X=X_{*}$ and $L_{*}=L \circ u$, then

$$
H H^{X}(L)=H H^{X_{*}}\left(L_{*}\right) .
$$

In case we take $X$ and $X_{*}$ to be a wedge of $n$ circles $\vee_{n} S^{1}$, the automorphism group $\operatorname{Aut}\left(F_{n}\right)$ acts on $\vee_{n} S^{1}$ up to homotopy by basepoint preserving maps and hence we obtain a representation of $\operatorname{Aut}\left(F_{n}\right)$ on $H H^{\vee_{n} S^{1}}\left(L_{*}\right)$. Similarly, the outer automorphism group $\operatorname{Out}\left(F_{n}\right)$ acts on $\vee_{n} S^{1}$ up to homotopy and hence we obtain a representation of $\operatorname{Out}\left(F_{n}\right)$ on $H H^{\vee_{n} S^{1}}(L)$. While this result should at least morally be known to experts, the representations of $\operatorname{Out}\left(F_{n}\right)$ arising in this manner seem to have received little attention in the literature. We will study a few special cases. The representations that we obtain inherit an additional filtration (the Hodge or Poincaré-Birkhoff-Witt filtration) such that the associated graded representation factors through $\operatorname{GL}(n, \mathbb{Z})$. We show that in general the representations of $\operatorname{Out}\left(F_{n}\right)$ thus obtained do not factor through $\operatorname{GL}(n, \mathbb{Z})$, but are nontrivial iterated extensions of $\mathrm{GL}(n, \mathbb{Z})$ representations.

\footnotetext{
${ }^{2}$ This definition can also be adjusted to realizations of any simplicial sets non-necessarily finite by using the right Kan extention of $L$ (respectively $L_{*}$ ) to the category of all (pointed) sets [28].

${ }^{3}$ This particular case of higher Hochschild homology is also called topological factorisation (or chiral) cohomology, see for example [3. 16].
} 
In particular, it is an open problem to determine the lowest dimensional representations of $\operatorname{Out}\left(F_{n}\right)$ that do not factor through $\operatorname{GL}(n, \mathbb{Z}) \sqrt{4}$ A lower bound has been obtained by D. Kielak [19], who showed that the dimension must be at least

$$
\left(\begin{array}{c}
n+1 \\
2
\end{array}\right)
$$

For $n=3$ the lower bound was refined to 7 (instead of 6) [20]. We obtain an upper bound as follows.

Theorem 1. For $n \geq 3$, the representations of $\operatorname{Out}\left(F_{n}\right)$ on $H H^{\vee_{n} S^{1}}(\mathbf{C}(\mathfrak{g}))$, where $\mathbf{C}(\mathfrak{g})$ is the Chevalley complex of a free Lie algebra $\mathfrak{g}=$ FreeLie $(x)$ in one generator $x$ of odd degree, contain a direct summand representation which does not factor through $\mathrm{GL}(n, \mathbb{Z})$ and has dimension

$$
\frac{n\left(n^{2}+5\right)}{6}
$$

In particular, for $n=3$ this representation saturates the lower bound 7 obtained in [20].

The previously known representations with such property have the smallest dimension 21 for $n=3$ and

$$
\left(2^{n}-1\right)\left(\begin{array}{c}
n-1 \\
2
\end{array}\right)
$$

for $n \geq 4$, see [19, Section 4], and also [5, 17].

The higher Hochschild homology on spheres was introduced and studied in the original work of Pirashvili [28] and on wedges of spheres it was studied in [30, 31] in connection with the homology and homotopy of spaces of higher dimensional string links. An interesting feature of this homology is that it admits a decomposition into a direct product, and the factors of this Hodge splitting depend only on the parity of the dimensions of the spheres. In particular, if we know $H H^{\vee_{n} S^{1}}\left(L_{*}\right)$ with the Hodge decomposition, we can reconstruct $H H^{\vee_{n} S^{d}}\left(L_{*}\right)$ for any other odd $d$. On the other hand, the homotopy type of a map $\vee_{n} S^{d} \rightarrow \vee_{n} S^{d}, d \geq 2$, is completely determined by the map in homology. Therefore, $H H^{\vee_{n} S^{d}}\left(L_{*}\right), d \geq 2$, is acted upon by the monoid $\operatorname{End}\left(\mathbb{Z}^{n}\right)$ of endomorphisms of $\mathbb{Z}^{n}$. For $d=1$, we get an action of the monoid $\operatorname{End}\left(F_{n}\right)$ of endomorphisms of a free group $F_{n}$. In Section 3 we define a certain explicit complex $C H^{\vee_{n} S^{1}}\left(L_{*}\right)$ computing $H H^{\vee_{n} S^{1}}\left(L_{*}\right)$.

Theorem 2. For any $\Gamma$-module $L_{*}$, the action of $\operatorname{End}\left(F_{n}\right)$ on $H H^{\vee_{n} S^{1}}\left(L_{*}\right)$ is naturally lifted on the level of the complex $\mathrm{CH}^{\vee_{n} S^{1}}\left(L_{*}\right)$. Moreover this action respects the Hodge splitting as an increasing filtration, and the action on the associated graded complex $\operatorname{grCH} \mathrm{V}^{\mathrm{V}{ }^{1}}\left(L_{*}\right)$ factors through $\operatorname{End}\left(\mathbb{Z}^{n}\right)$.

We will see in Section 4 that as an $\operatorname{End}\left(\mathbb{Z}^{n}\right)$ module, $g r H H^{\vee_{n} S^{1}}\left(L_{*}\right)$ is (up to regrading) naturally isomorphic to $H H^{\vee_{n} S^{d}}\left(L_{*}\right)$ for any odd $d \geq 3$.

The fact that the $\operatorname{End}\left(F_{n}\right)$ action above respects the Hodge filtration is actually a manifestation of a more general phenomenon. We show in Section 4 that the Hodge filtration in $H H^{X_{*}}\left(L_{*}\right)$, that can also be called PoincaréBirkhoff-Witt filtration, is defined functorially in $X_{*}$ and $L_{*}$. This filtration is an interesting phenomenon in itself that does not seem to appear earlier in any kind of functor calculus. In particular, the Hodge filtration should not be confused with the cardinality or rank (co)filtration considered, for example, in [3, 18], and inspired from the manifold functor calculus [35], see Subsection 4.3. In that subsection we also explain in which sense the Hodge filtration in the Hochschild-Pirashvili homology on suspensions is exhaustive: it is dense in the topology induced by the cardinality cofiltration.

Theorem 2] can be "categorified" to all suspensions and maps between them. More specifically, let Top denote the category of pointed topological spaces with morphisms homotopy classes of pointed maps. Let Top $\left.\right|_{\Sigma}$ denote its full subcategory whose objects are suspensions. By $\Sigma\left(\mathrm{Top}_{*}\right)$ we denote the image category of the suspension functor $\Sigma:$ Top $_{*} \rightarrow \mathrm{Top}_{*}$. Notice that any suspension is rationally equivalent to a wedge of spheres [9, Theorem 24.5]. Thus, for the sake of concreteness and slightly simplifying the matters, the reader can think about the category Top $\left.\right|_{*}$ as about the full subcategory in $\mathrm{Top}_{*}$ of wedges of spheres of possibly different dimensions $\geq 1$. The following theorems generalize Theorem 2 on this category $\operatorname{Top}_{*} \mid \Sigma$.

Theorem 3. For any right $\Gamma$-module $L_{*}$, the cofunctor $H H^{(-)}\left(L_{*}\right): \operatorname{Top}_{*}{ }^{o p} \rightarrow g$ Vect admits an increasing filtration generalizing the Hodge filtration on $\mathrm{HH}^{\vee_{n} S^{1}}\left(L_{*}\right)$, such that the completed associated graded functor gr $H^{(-)}\left(L_{*}\right)$ restricted on $\left.\mathrm{Top}_{*}\right|_{\Sigma}$ factors through the reduced homology functor $\tilde{H}_{*}: \operatorname{Top}_{*} \rightarrow$ gVect. Over $\Sigma\left(\operatorname{Top}_{*}\right)$, this filtration splits in the sense that one has a natural isomorphism $\left.\left.H^{(-)}\left(L_{*}\right)\right|_{\Sigma\left(\operatorname{Top}_{*}\right)} \rightarrow \operatorname{gr} H H^{(-)}\left(L_{*}\right)\right|_{\Sigma\left(\operatorname{Top}_{*}\right)}$.

In Section 5 we construct a cofunctor $\mathrm{CH}^{(-)}\left(L_{*}\right):\left(\left.\operatorname{Top}_{*}\right|_{\Sigma}\right)^{o p} \rightarrow$ dgVect.

Theorem 4. The cofunctor $\mathrm{CH}^{(-)}\left(L_{*}\right):\left(\left.\operatorname{Top}_{*}\right|_{\Sigma}\right)^{o p} \rightarrow$ dgVect has the following properties

\footnotetext{
${ }^{4}$ One assumes $n \geq 3$ as $\operatorname{Out}\left(F_{2}\right)=\mathrm{GL}(2, \mathrm{Z})$.
} 
- $H_{*} \circ \mathrm{CH}^{(-)}\left(L_{*}\right)=H H^{(-)}\left(L_{*}\right)$.

- The complex $\mathrm{CH}^{\vee_{n} S^{1}}\left(L_{*}\right)$ is identical to $C H^{\vee_{n} S^{1}}\left(L_{*}\right)$.

- This functor admits an increasing (Hodge) filtration compatible with the Hodge filtration in homology.

- The completed associated graded functor $g r \mathrm{CH}^{(-)}\left(L_{*}\right)$ factors through the reduced homology finctor $\tilde{H}_{*}: \mathrm{Top}_{*} \mid \Sigma \rightarrow$ gVect.

- Over $\Sigma\left(\operatorname{Top}_{*}\right)$, the Hodge filtration in $\mathrm{CH}^{(-)}\left(L_{*}\right)$ splits in the sense that one has a natural isomorphism $\left.\left.\mathrm{CH}^{(-)}\left(L_{*}\right)\right|_{\Sigma\left(\mathrm{Top}_{*}\right)} \rightarrow \operatorname{gr} \mathrm{CH}^{(-)}\left(L_{*}\right)\right|_{\Sigma\left(\mathrm{Top}_{*}\right)}$.

More concretely when we say that the functors $g r H H^{(-)}\left(L_{*}\right):\left.\operatorname{Top}_{*}\right|_{\Sigma} \rightarrow g V e c t$ and $g r \mathrm{CH}^{(-)}\left(L_{*}\right):\left.\operatorname{Top}_{*}\right|_{\Sigma} \rightarrow$ dgVect factor through $\tilde{H}_{*}:\left.\operatorname{Top}_{*}\right|_{\Sigma} \rightarrow$ gVect we mean that for any pointed space $Y_{*}$, both $\operatorname{gr} H H^{\Sigma Y_{*}}\left(L_{*}\right)$ and $g r \mathrm{CH}^{\Sigma Y_{*}}\left(L_{*}\right)$ can be described as a power series expression in $\tilde{H}_{*} \Sigma Y_{*}$ :

$$
\begin{aligned}
\operatorname{grHH}^{\Sigma Y_{*}}\left(L_{*}\right) & =\prod_{n} \operatorname{Hom}_{n}\left(\left(\tilde{H}_{*} \Sigma Y_{*}\right)^{\otimes n}, \mathcal{H}_{L_{*}}(n)\right), \\
\operatorname{grCH}^{\Sigma Y_{*}}\left(L_{*}\right) & =\prod_{n} \operatorname{Hom}_{n}\left(\left(\tilde{H}_{*} \Sigma Y_{*}\right)^{\otimes n}, C_{L_{*}}(n)\right),
\end{aligned}
$$

where $C_{L_{*}}$ is some symmetric sequence in chain complexes depending on $L_{*}$, and $\mathcal{H}_{L_{*}}$ is its homology symmetric sequence. The fact that the Hodge filtration splits over $\Sigma\left(\operatorname{Top}_{*}\right)$ means that we have isomorphisms

$$
\begin{aligned}
& \mathrm{CH}^{\Sigma Y_{*}}\left(L_{*}\right) \stackrel{\simeq}{\longrightarrow} \operatorname{grCH} \mathrm{CH}^{\Sigma Y_{*}}\left(L_{*}\right), \\
& H H^{\Sigma Y_{*}}\left(L_{*}\right) \stackrel{\simeq}{\longrightarrow} g r H H^{\Sigma Y_{*}}\left(L_{*}\right)
\end{aligned}
$$

natural in $\Sigma Y_{*} \in \Sigma\left(\operatorname{Top}_{*}\right)$. The $n$-th term of the Hodge splitting is exactly the $n$-th factor in (2) and (3). (This splitting also means that the higher Hochschild complexes for suspensions split as a product of complexes.) In case a pointed map $f: \Sigma Y_{*} \rightarrow \Sigma Z_{*}$ is not a suspension, the Hodge splitting in the higher Hochschild complexes/homology (via isomorphisms (4)-(5)) behaves like a filtration: higher terms of the splitting can be send non-trivially to lower ones. In Section 6 we compute how from the given rational homotopy type of a map of suspensions one gets the induced map between the terms of the splitting. We also demonstrate this on some examples, such as the Hopf map $S^{3} \rightarrow S^{2}$ and a non-trivial pointed map $S^{2} \rightarrow S^{2} \vee S^{1}$.

Some of the techniques that we develop for suspensions work equally well for general spaces. In Section 7 we briefly consider this general case of non-suspensions. Theorems 5,6 and Proposition 7.4 describe these more general higher Hochschild complexes in the case $L_{*}=M \otimes C^{\otimes \bullet}$ as some kind of homotopy base change type of Chevalley complexes. In this section we also show that for a connected pointed space $X_{*}$ (of finite type) the Hodge filtration splits for any coefficient $\Gamma$ module $L_{*}$ if and only if $X_{*}$ is rationally homology equivalent to a suspension.

Acknowledgements. We thank G. Arone, B. Fresse, G. Ginot, and D. Kielak for helpful discussions. V.T. thanks the MPIM and the IHES, where he spent his sabbatical and where he started to work on this project. T.W. has been partially supported by the Swiss National Science foundation, grant 200021_150012, and the SwissMAP NCCR funded by the Swiss National Science foundation.

\section{Special case of $\operatorname{End}\left(F_{n}\right)$ action}

In this section we look at the special case $L_{*}=M \otimes C^{\otimes \bullet}$, where $C$ is a cocommutative coalgebra and $M$ a $C$-comodule as before. If not otherwise stated we will always assume that $C$ is simply connected. We will define a complex $C H^{\vee_{n} S^{1}}\left(M \otimes C^{\otimes \bullet}\right)$ and an $\operatorname{End}\left(F_{n}\right)$ action on it. In Section 3 we explain why this complex computes $H H^{\vee_{n} S^{1}}\left(M \otimes C^{\otimes \bullet}\right)=H H^{\vee_{n} S^{1}}(C, M)$ and why the $\operatorname{End}\left(F_{n}\right)$ action that we define corresponds to the topological action. Define $C H^{\vee_{n} S^{1}}\left(M \otimes C^{\otimes \bullet}\right)$ as $M \otimes(\Omega C)^{\otimes n}$, where $\Omega C$ is the cobar construction of $C$ - as a space it is a free associative algebra generated by $C[1]$. The differential

$$
d=d_{M}+d_{C}+\delta
$$

where $d_{M}$ and $d_{C}$ are induced by the differentials on $M$ and $\Omega C$ respectively and

$$
\delta\left(m \otimes b_{1} \otimes \ldots \otimes b_{n}\right)=\sum \sum_{j} \pm m^{\prime} \otimes \ldots \otimes\left[m^{\prime \prime}, b_{j}\right] \otimes \ldots \otimes b_{n},
$$

where we used Sweedler's notation; \pm is the Koszul sign due to permutation of $m^{\prime \prime}$ with $b_{i}$ 's.

We can assume without loss of generality that $C=\mathbf{C}(g)$ is the Chevalley complex of a dg Lie algebra $g$ concentrated in strictly positive degrees. (If not, take for $\mathfrak{g}$ the Harrison complex of $C$.) As a cocommutative coalgebra it is freely cogenerated by $\mathrm{g}[-1]$. In the latter case the aforementioned complex is quasi-isomorphic to $M \otimes\left(\mathcal{U}_{\mathfrak{g}}\right)^{\otimes n}$, where $\mathcal{U}_{\mathfrak{g}}$ is the universal envelopping algebra of $\mathfrak{g}$, with differential

$$
d=d_{M}+d_{\mathfrak{g}}+\delta
$$


defined similarly: $d_{M}$ and $d_{\mathfrak{g}}$ are induced from the differentials on $M$ and $\mathfrak{g}$ and

$$
\delta\left(m \otimes b_{1}, \ldots, b_{n}\right)=\sum \sum_{j} \pm m^{\prime} \otimes b_{1} \otimes \ldots \otimes\left[\pi\left(m^{\prime \prime}\right), b_{j}\right] \otimes \cdots \otimes b_{n},
$$

where $\pi: \mathbf{C}(\mathfrak{g}) \rightarrow \mathfrak{g}$ is the projection to the cogenerators.

The action of $\operatorname{End}\left(F_{n}\right)$ on $M \otimes(\mathcal{U} \mathfrak{g})^{\otimes n}$ and $M \otimes(\Omega C)^{\otimes n}$ is described by the same formulas. Both $\mathcal{U} \mathfrak{g}$ and $\Omega C$ are cocommutative Hopf algebras. In Sweedler's notation the iterated coproduct is written as

$$
\Delta^{k} b=\sum b^{(1)} \otimes b^{(2)} \otimes \ldots \otimes b^{(k)} .
$$

Since the coproduct is cocommutative, we will be writing instead

$$
\Delta^{k} b=\sum b^{(\bullet)} \otimes b^{(\bullet)} \otimes \ldots \otimes b^{(\bullet)} .
$$

Let $\Psi \in \operatorname{End}\left(F_{n}\right)$ send

$$
x_{i} \mapsto x_{\alpha_{i 1}}^{\varepsilon_{i 1}} \cdot x_{\alpha_{i 2}}^{\varepsilon_{i 2}} \cdot \ldots \cdot x_{\alpha_{i k_{i}}}^{\varepsilon_{k_{i}}}, \quad i=1 \ldots n,
$$

where $\varepsilon_{i j}= \pm 1, \alpha_{i j} \in\{1 \ldots n\}$. We let $\beta_{i j}=\frac{1-\varepsilon_{i j}}{2} \in\{0,1\}$ and define

$$
\Psi^{*}\left(m \otimes b_{1} \otimes \ldots \otimes b_{n}\right):=m \otimes \sum \pm \bigotimes_{i=1}^{n} \prod_{j=1}^{k_{i}} s^{\beta_{i j}}\left(b_{\alpha_{i j}}^{(\bullet)}\right),
$$

where the sign \pm is the Koszul sign arising from the factors permutations, $s$ is the antipod.

Example 1.1. (a) $n=1 ; x_{1} \mapsto\left(x_{1}\right)^{2}$.

(b) $n=1 ; x_{1} \mapsto x_{1}^{-1}$.

$$
\Psi^{*}(m \otimes b)=m \otimes \sum b^{\prime} \cdot b^{\prime \prime} .
$$

$$
\Psi^{*}(m \otimes b)=m \otimes s(b) .
$$

(c) $n=2 ; x_{1} \mapsto x_{1} \cdot x_{2}, x_{2} \mapsto x_{2}$.

$$
\Psi^{*}\left(m \otimes b_{1} \otimes b_{2}\right)=m \otimes \sum b_{1} \cdot b_{2}^{\prime} \otimes b_{2}^{\prime \prime} .
$$

Proposition 1.2. The formula (9) defines the right action of $\operatorname{End}\left(F_{n}\right)$ on the complexes $M \otimes\left(\mathcal{U}_{\mathfrak{g}}\right)^{\otimes n}$ and $M \otimes(\Omega C)^{\otimes n}$.

Proof. To see that $\Psi^{*}$ is a morphism of complexes we notice that it commutes with each term of the differentials (6) and (7): it commutes with $d_{M}$ by obvious reasons; it commutes with $d_{\mathfrak{g}}$ since both product and coproduct of $\mathcal{U}_{\mathfrak{g}}$ are morphisms of complexes; it commutes with $\delta$ since both product and coproduct respect the $g$ action.

For the composition, it is quite easy to see that $\left(\Psi_{1} \circ \Psi_{2}\right)^{*}=\Psi_{2}^{*} \circ \Psi_{1}^{*}$, where the composition $\Psi_{1} \circ \Psi_{2}$ is understood as substitution without simplification. We only need to check that in case $\left(\Psi_{1} \circ \Psi_{2}\right)\left(x_{i}\right)$ has two consecutive factors $x_{j}$ and $x_{j}^{-1}$ for some $i$, then $\left(\Psi_{1} \circ \Psi_{2}\right)^{*}$ is the same as if these factors are cancelled out. But in such case, $\left(\Psi_{1} \circ \Psi_{2}\right)^{*}\left(m \otimes b_{1} \otimes \ldots \otimes b_{n}\right)$ also has two consecutive factors $b_{j}^{(\bullet)}$ and $s\left(b_{j}^{(\bullet)}\right)$, which can also be eliminated:

$$
\sum b_{j}^{(\bullet)} \cdot s\left(b_{j}^{(\bullet)}\right) \otimes\left(b_{j}^{(\bullet)}\right)^{\otimes k}=1 \otimes \sum\left(b_{j}^{(\bullet)}\right)^{\otimes k}=1 \otimes \Delta^{k} b_{j}=\sum s\left(b_{j}^{(\bullet)}\right) \cdot b_{j}^{(\bullet)} \otimes\left(b_{j}^{(\bullet)}\right)^{\otimes k}
$$

1.1. Hodge decomposition/filtration. The Poincaré-Birkhoff-Witt isomorphism $S \mathfrak{g} \rightarrow \mathcal{U} \mathfrak{g}$ respects both the coalgebra and $\mathfrak{g}$ action structures. As a corollary, the induced map

$$
M \otimes(S \mathfrak{g})^{\otimes n} \rightarrow M \otimes(\mathcal{U g})^{\otimes n}
$$

is an isomorphism of complexes. The image of the subcomplex $M \otimes S^{m_{1}} \mathfrak{g} \otimes \ldots \otimes S^{m_{n}} \mathfrak{g}$ in $M \otimes(\mathcal{U} \mathfrak{g})^{\otimes n}$ is called $\left(m_{1}, \ldots, m_{n}\right)$ Hodge multidegree component, whose total Hodge degree is $m=m_{1}+\ldots+m_{n}$. One has

$$
\bigoplus_{m_{1}+\ldots+m_{n}=m} M \otimes S^{m_{1}} \mathfrak{g} \otimes \ldots \otimes S^{m_{n}} \mathfrak{g}=M \otimes S^{m}\left(H^{1} \otimes \mathfrak{g}\right)
$$

where $H^{1}:=H^{1}\left(\vee_{n} S^{1}, \mathbb{Z}\right)=\mathbb{Z}^{n}$ viewed as a space concentrated in degree zero. Below $H_{1}:=H_{1}\left(\vee_{n} S^{1}, \mathbb{Z}\right)$.

Proposition 1.3. The action of $\operatorname{End}\left(F_{n}\right)$ on $M \otimes(\mathcal{U})^{\otimes n}$ preserves the total Hodge degree as a filtration. The induced action on the associated graded complex $\operatorname{gr} M \otimes(\mathcal{U} \mathfrak{g})^{\otimes n}$ factors through $\operatorname{End}\left(H_{1}\right)=\operatorname{End}\left(\mathbb{Z}^{n}\right)$ as one has

$$
\operatorname{gr} M \otimes(\mathcal{U} \mathfrak{g})^{\otimes n}=M \otimes S\left(H^{1} \otimes \mathfrak{g}\right) .
$$

This proposition is a particular case of Theorem 2 . 
Proof. The Hodge filtration is preserved because both the product and coproduct of $\mathcal{U}_{\mathfrak{g}}$ preserve the PoincaréBirkhoff-Witt filtration. Notice also that if we apply (9) to define an $\operatorname{End}\left(F_{n}\right)$ action on $M \otimes(S \mathfrak{g})^{\otimes n}$, we get exactly $M \otimes(S \mathfrak{g})^{\otimes n} \simeq M \otimes S\left(H^{1} \otimes \mathfrak{g}\right)$ as a right $\operatorname{End}\left(F_{n}\right)$ module.

Remark 1.4. It will be shown in Subsection 4.1 (see Remark 4.6) that for any pointed space $Y_{*}$ of finite type, the Hochschild-Pirashvili homology $H H^{\Sigma Y_{*}}(C(\mathfrak{g}), M)$ is computed by the complex

$$
M \otimes S\left(\tilde{H}^{*} Y_{*} \otimes \mathfrak{g}\right),
$$

where $\tilde{H}^{*}(Y)$ is the reduced cohomology of $Y$ viewed as a negatively graded vector space. The differential has the same form (7) 5

\section{2. $\operatorname{Out}\left(F_{n}\right)$ Representations. Proof of Theorem 1}

Recall isomorphism (1), which in particular implies that in case $M=C$ the action of $\operatorname{Aut}\left(F_{n}\right)$ on $H H^{\vee_{n} S^{1}}(C, M)=$ $H H^{\vee_{n} S^{1}}(C)$ descends to an $\operatorname{Out}\left(F_{n}\right)$ action. Recall also that according to Proposition 1.3 the higher Hochschild homology $H H^{\vee_{n} S^{1}}(C, M)$ carries a Hodge filtration such that the action of $\operatorname{Aut}\left(F_{n}\right)$ on the associated graded factors through $\operatorname{GL}(n, \mathbb{Z})$. In other words, all $\operatorname{Aut}\left(F_{n}\right)$ and $\operatorname{Out}\left(F_{n}\right)$ modules obtained in this manner can be obtained by iterated extension of $\mathrm{GL}(n, \mathbb{Z})$-modules by $\operatorname{GL}(n, \mathbb{Z})$-modules.

2.1. Example 1: Polynomial coalgebras. If $C=\mathbb{Q}\left[x_{1}, \ldots, x_{n}\right]$ is a cofree cocommutative coalgebra (in potentially odd generators), we have $\mathfrak{g}=\xi_{1} \mathbb{Q} \oplus \cdots \oplus \xi_{n} \mathbb{Q}$ as abelian Lie algebra, where the generators $\xi_{j}$ are degree shifted by one unit with respect to the generators $x_{j}$. In this case the Hodge grading is preserved by the $\operatorname{Aut}\left(F_{n}\right)$ action (because $\mathcal{U}_{\mathfrak{g}}$ is commutative) and hence all representations obtained factor through $\operatorname{GL}(n, \mathbb{Z})$. Since the differential on $C \otimes(\mathcal{U} \mathfrak{g})^{\otimes n}$ vanishes the higher Hochschild homology is just

$$
H H^{\vee_{n} S^{1}}(C) \cong C \otimes S\left(H^{1} \otimes \mathfrak{g}\right)
$$

with the $\operatorname{Out}\left(F_{n}\right)$ action factoring through $G L(n, \mathbb{Z})=G L\left(H_{1}\right)$, which acts by the standard action on $\mathbb{Z}^{n}=H^{1}$.

2.2. Example 2: Dual numbers. Consider the coalgebra of dual numbers $\mathbb{Q} \oplus x \mathbb{Q}$, where $x$ is a primitive cogenerator of even degree. The (Koszul) dual Lie algebra is the free Lie algebra in one odd generator $\xi$, i.e., $\mathfrak{g}=\xi \mathbb{Q} \oplus[\xi, \xi] \mathbb{Q}$. Then the associated graded of $C H^{\vee_{n} S^{1}}\left(C \otimes C^{\otimes \bullet}\right)$ may be identified with

$$
\operatorname{grC} H^{\vee_{n} S^{1}}\left(C \otimes C^{\otimes \bullet}\right) \cong C \otimes S\left(H^{1} \otimes \mathfrak{g}\right) \cong C \otimes \mathbb{Q}\left[\xi_{1}, \ldots, \xi_{n}, \eta_{1}, \ldots, \eta_{n}\right] .
$$

Here $\xi_{j}$ corresponds to $\xi$ on the $j$-th circle and $\eta_{j}$ corresponds to $\eta=[\xi, \xi]=2 \xi^{2}$ on the $j$-th circle. Notice that $a d_{\xi}(\xi)=\eta$ and $a d_{\xi}(\eta)=0$. The complex has length 2 :

$$
0 \leftarrow 1 \otimes \mathbb{Q}\left[\xi_{1}, \ldots, \xi_{n}, \eta_{1}, \ldots, \eta_{n}\right] \stackrel{d}{\longleftarrow} x \otimes \mathbb{Q}\left[\xi_{1}, \ldots, \xi_{n}, \eta_{1}, \ldots, \eta_{n}\right] \leftarrow 0 .
$$

The differential is defined such that

$$
\begin{aligned}
d\left(x \otimes P\left(\xi_{1}, \ldots, \xi_{n}, \eta_{1}, \ldots, \eta_{n}\right)\right)=\sum_{j=1}^{n} 1 \otimes a d_{\xi_{j}} P\left(\xi_{1}, \ldots, \xi_{n}, \eta_{1}, \ldots, \eta_{n}\right) & \\
& =\sum_{j=1}^{n} 1 \otimes \eta_{j} \frac{\partial}{\partial \xi_{j}} P\left(\xi_{1}, \ldots, \xi_{n}, \eta_{1}, \ldots, \eta_{n}\right)
\end{aligned}
$$

The differential can be identified with the de Rham differential on an $n$-dimensional odd vector space, identifying $\eta_{j}$ with $d_{d R} \xi_{j}$. One can identify the corresponding representations of $\operatorname{GL}(n, \mathbb{Z})$. Namely, if we fix in the associated graded the Hodge degree to be $m$, then the corresponding representations of GL $(n, \mathbb{Z})$ one obtains correspond to partitions of the form $m=\ell+1+\cdots+1$. To be precise the homology is the sum $U^{I} \oplus U^{I I}$, where $U^{I}=\operatorname{coker} d$, $U^{I I}=\operatorname{ker} d$. The part of degree $k$ in $\xi$ and $\ell$ in $\eta$ is sent by $d$ to the part of degree $k-1$ in $\xi$ and $\ell+1$ in $\eta$ :

$$
0 \leftarrow \Lambda^{k-1} H^{1} \otimes S^{\ell+1} H^{1} \stackrel{d}{\longleftarrow} \Lambda^{k} H^{1} \otimes S^{\ell} H^{1} \leftarrow 0 .
$$

The GL $(n)$ module $\Lambda^{k} H^{1} \otimes S^{\ell} H^{1}$ is a direct sum of 2 representations encoded by partitions $(\ell+k)=\ell+1+\ldots+1$ and $(\ell+k)=(\ell+1)+1+\ldots+1$. We conclude that the kernel of $d$ in this bigrading is $V_{\left(\ell, 1^{k}\right)}$ and the cokernel of $d$ is $V_{\left(\ell+2,1^{k-2}\right)}$. The bigrading by $\xi$ and $\eta$ is preserved in $C \otimes(\mathcal{U} \mathfrak{g})^{\otimes n}$ only as a filtration. Instead one can consider

\footnotetext{
${ }^{5}$ Unless certain convergency properties are satisfied, $S(-)$ should be undersood as a completed symmetric algebra, i.e. a direct product $\prod_{m \geq 0} S^{m}(-)$ rather than a direct sum. Similarly the tensor product should be understood as the completed tensor product with respect to the homological degree of $\mathfrak{g}$.
} 
the total $\xi$ grading by assigning 1 to each $\xi$ and 2 to each $\eta=[\xi, \xi]$. The component $U_{N}^{I} \oplus U_{N}^{I I}$ in the homology of total $\xi$ degree $N$ is a filtered space, whose associated graded is

$$
g r U_{N}^{I}=\bigoplus_{2 \ell+k=N+1} V_{\left(\ell, 1^{k}\right)}, \quad g r U_{N}^{I I}=\bigoplus_{2 \ell+k=N} V_{\left(\ell, 1^{k}\right)}
$$

For both $U^{I}$ and $U^{I I}$ the Hodge degree of $V_{\left(\ell, 1^{k}\right)}$ is $\ell+k$.

2.3. The lowest non-trivial example worked out. Let us consider the first $\operatorname{Out}\left(F_{n}\right)$ representation obtained by the above methods that does not factor through GL $(n, \mathbb{Z})$. It is obtained as the cokernel of the differential in the dual numbers example above for $n=3$ and the total $\xi$ degree 3. It was denoted by $U_{3}^{I}$ in the previous subsection. The representation is 7 dimensional. As in Subsection 2.2 one sees that the associated graded representation splits into two $\operatorname{GL}(3, \mathbb{Z})$ representations

$$
g r U_{3}^{I}=V_{(2)} \oplus V_{(1,1,1)}
$$

In other words, $U_{3}^{I}$ is an extension

$$
0 \rightarrow V_{(2)} \rightarrow U_{3}^{I} \rightarrow V_{(1,1,1)} \rightarrow 0 .
$$

A representative of the cohomology class in $H C^{\vee_{3} S^{1}}(C)$ spanning the $V_{(1,1,1)}$ part is

$$
e:=1 \otimes \xi \otimes \xi \otimes \xi
$$

Representatives forming a basis of $V_{(2)}$ are

$$
\begin{array}{ll}
f_{1}:=1 \otimes[\xi, \xi] \otimes \xi \otimes 1 \cong-1 \otimes \xi \otimes[\xi, \xi] \otimes 1 & f_{2}:=1 \otimes[\xi, \xi] \otimes 1 \otimes \xi \\
f_{3}:=1 \otimes 1 \otimes[\xi, \xi] \otimes \xi & f_{4}:=1 \otimes[\xi, \xi] \xi \otimes 1 \otimes 1 \\
f_{5}:=1 \otimes 1 \otimes[\xi, \xi] \xi \otimes 1 & f_{6}:=1 \otimes 1 \otimes 1 \otimes[\xi, \xi] \xi
\end{array}
$$

2.4. The proof of Theorem 1 More generally let us consider representation $U_{3}^{I}$ of $\operatorname{Out}\left(F_{n}\right)$ for arbitrary $n \geq 3$. We claim that this representation satisfies the requirements of Theorem 1 i.e., it does not factor through $\operatorname{GL}(n, \mathbb{Z})$ and it has dimension $\frac{n\left(n^{2}+5\right)}{6}$.

Indeed, as in Subsection 2.3 we can identify the associated graded representation under the Hodge filtration with

$$
g r U_{3}^{I}=V_{(2)} \oplus V_{(1,1,1)}
$$

where $V_{(2)}$ and $V_{(1,1,1)}$ are the irreducible representations of the linear group GL( $\left.n\right)$ corresponding to the partitions (2) and $(1+1+1)$. Hence we find that indeed

$$
\operatorname{dim} U_{3}^{I}=\operatorname{dim} V_{(2)}+\operatorname{dim} V_{(1,1,1)}=\frac{n(n+1)}{2}+\left(\begin{array}{l}
n \\
3
\end{array}\right)=\frac{n\left(n^{2}+5\right)}{6} .
$$

Next, we check that the representation does not factor through $\operatorname{GL}(n)$. Consider $E_{12}, E_{\overline{1} \overline{2}} \in \operatorname{Out}\left(F_{n}\right)$ that send

$$
E_{12}\left(x_{i}\right)=\left\{\begin{array}{ll}
x_{1} x_{2}, & i=1 ; \\
x_{i}, & \text { otherwise; }
\end{array} \quad E_{\overline{1} \overline{2}}\left(x_{i}\right)= \begin{cases}x_{2} x_{1}, & i=1 ; \\
x_{i}, & \text { otherwise }\end{cases}\right.
$$

We will show that the action of $E_{12}$ is different from that of $E_{\overline{1} \overline{2}}$ in the representation $U_{3}^{I}$ for $n \geq 3$. Indeed, choosing basis vectors as in Subsection 2.3 we find that

$$
E_{12} \cdot(1, \xi, \xi, \xi, 1, \ldots, 1)=(1, \xi, \xi, \xi, 1, \ldots, 1)+\frac{1}{2}(1,[\xi, \xi], 1, \xi, 1, \ldots, 1)
$$

while

$$
E_{\overline{1} \overline{2}} \cdot(1, \xi, \xi, \xi, 1, \ldots, 1)=(1, \xi, \xi, \xi, 1, \ldots, 1)-\frac{1}{2}(1,[\xi, \xi], 1, \xi, 1, \ldots, 1) .
$$

To recall $U_{3}^{I}$ is the cokernel of $d$. Thus we need to verify that $(1,[\xi, \xi], 1, \xi, 1, \ldots, 1) \in \mathbb{Q} \otimes(S \mathfrak{g})^{\otimes n}$ is not in the image of $d$. As we have seen in Subsection 2.2 $d$ is the de Rham differential which is acyclic on non-constant polynomials in $\xi_{i}$ and $\eta_{j}$, thus we only have to check that the corresponding polynomial is not de Rham closed:

$$
\sum_{j=1}^{n} \eta_{j} \frac{\partial}{\partial \xi_{j}}\left(\eta_{1} \xi_{3}\right)=\eta_{1} \eta_{3} \neq 0
$$


2.5. Bead representations. Generalizing the example of dual numbers we may consider the coalgebra

$$
C_{N}=\mathbb{Q} \oplus x_{1} \mathbb{Q} \oplus x_{2} \mathbb{Q} \oplus \ldots \oplus x_{N} \mathbb{Q},
$$

where the cogenerators $x_{i}$ are of even degrees and primitive. The Koszul dual Lie algebra is again free

$$
\mathfrak{g}=\operatorname{FreeLie}\left(\xi_{1}, \ldots, \xi_{N}\right) .
$$

There is a $\mathbb{Z}^{N}$ grading on $C_{N}$ and a representation of $\mathbb{S}_{N}$, and hence a similar grading and action on the higher Hochschild homology $H H^{\vee_{n} S^{1}}\left(C_{N}\right)$. We may introduce a representation of $\operatorname{Out}\left(F_{n}\right)$ for every irreducible representation $V_{\lambda}$ of $\mathbb{S}_{N}$ labelled by a partition $\lambda$ of $N$ :

$$
U_{\lambda}=H H^{\vee_{n} S^{1}}\left(C_{N}\right)^{1, \ldots, 1} \otimes{ }_{N} V_{\lambda}
$$

Here the superscript $(\cdot)^{1, \ldots, 1}$ shall mean that we pick out the piece of $\mathbb{Z}^{N}$-degree $(1, \ldots, 1)$. We will call $U_{\lambda}$ the bead representation 6 of $\operatorname{Out}\left(F_{n}\right)$ associated to the partition $\lambda$. Notice that the obtained complex is again of length 2 . Thus we have again $U_{\lambda}=U_{\lambda}^{I} \oplus U_{\lambda}^{I I}$ where $U_{\lambda}^{I}$ is the cokernel of the differential and $U_{\lambda}^{I I}$ is the kernel. We will call $U_{\lambda}^{I}$ the bead representation of first type and $U_{\lambda}^{I I}$ the bead representation of second type. $]^{7}$

Open problem: Describe $U_{\lambda}$. In particular, what are the dimensions $\operatorname{dim}\left(U_{\lambda}^{I I I}\right)$ ? If we decompose the associated graded $g r U_{\lambda}$ into irreducible representations of $\operatorname{GL}(n, \mathbb{Z})($ actually $\operatorname{GL}(n, \mathbb{R}))$

$$
g r U_{\lambda} \cong \oplus_{\mu} V_{\mu}
$$

which partitions $\mu$ occur in the direct sum, with what multiplicity?

\section{Complexes $C H^{\vee_{n} S^{1}}\left(L_{*}\right)$. Proof of Theorem 2}

Recall that in case the space $X$ (respectively pointed space $X_{*}$ ) is obtained as a realization of a (pointed) finite simplicial set $\mathcal{X}_{0}: \Delta^{o p} \rightarrow$ Fin (respectively $\mathcal{X}_{\bullet}: \Delta^{o p} \rightarrow \Gamma$ ), the higher Hochschild homology $H H^{X}(L)$ (respectively $H H^{X_{*}}\left(L_{*}\right)$ ) can be computed as the homology of the totalization of the cosimplicial chain complex $L \circ \mathcal{X}: \Delta \rightarrow d g$ Vect (respectively $L_{*} \circ \mathcal{X}_{*}: \Delta \rightarrow d g V e c t$ ). The same construction works for realizations of bisimplicial (and more generally multisimplicial) sets. Indeed, if $X_{\text {.. }}$ is a bisimplicial set, then its realization $\left|X_{\text {.. }}\right|$ is homeomorphic to the realization $\left|\operatorname{diag}\left(\mathcal{X}_{\text {.o }}\right)\right|$ of its diagonal simplicial set. On the other hand, one also has the Eilenberg-Zilber quasi-isomorphism

$$
\operatorname{Tot}\left(\operatorname{diag} L \circ X_{\text {.. }} \stackrel{E Z}{\longrightarrow} \operatorname{Tot}\left(L \circ X_{\text {.. }}\right) .\right.
$$

As the first complex computes the Hochschild-Pirashvili homology of $\left|\operatorname{diag}\left(X_{\bullet . .}\right)\right|=\left|X_{. .}\right|$, so does the second.

Now notice that the complexes $M \otimes(\Omega C)^{\otimes n}$ can be obtained as totalization of an $n$-multicosimplicial chain complex (rather than just cosimplicial). (In fact its diagonal totalization is $M \otimes \Omega\left(C^{\otimes n}\right)$.) The corresponding multicosimplicial complex is obtained as the composition of $M \otimes C^{\otimes \bullet}$ with an $n$-multisimplicial model of $\vee_{n} S^{1}$. Let $S_{\text {. }}^{1}$ denote the standard simplicial model for $S^{1}$ : its set of $k$-simplices consists of a basepoint $*$ and also all monotonic non-constant sequences of 0's and 1's of length $k+1$. This set can be identified with $k_{*}$ (where $i \in k_{*}$ corresponds to a sequence with $i$ 1's). The $n$-multisimplicial model for $\vee_{n} S^{1}$, we denote it by $\left(\vee_{n} S^{1}\right) \underbrace{\bullet}_{n}$, is obtained as a degreewise wedge of $n n$-multisimplical sets. The $i$-th summand of the wedge is the product of $S$. and $(n-1)$ constant one-point simplicial sets, with $S_{\bullet}^{1}$ appearing on the $i$-th place in the product. Notice that the $\left(k_{1}, k_{2}, \ldots, k_{n}\right)$ component of $\left(\vee_{n} S^{1}\right) \underbrace{\ldots \bullet}_{n}$ is the set $\bigvee_{i=1}^{n}\left(k_{i}\right)_{*} \simeq\left(k_{1}+\ldots+k_{n}\right)_{*}$. Thus the totalization of our multicosimplial complex is

$$
C H^{\vee_{n} S^{1}}\left(L_{*}\right):=\operatorname{Tot}(L_{*} \circ\left(\vee_{n} S^{1}\right) \underbrace{\bullet \ldots \bullet}_{n})=\left(\prod_{\left(k_{1}, \ldots, k_{n}\right)} N L_{*}\left(\Sigma_{i=1}^{n} k_{i}\right)\left[\Sigma_{i=1}^{n} k_{i}\right], d=d_{1}+\ldots+d_{n}\right),
$$

where

$$
N L_{*}(k)=\bigcap_{i=1}^{k} \operatorname{ker} s_{i}^{*},
$$

and $s_{i}^{*}: L\left(k_{*}\right) \rightarrow L\left(k_{*} \backslash\{i\}\right)$ is the map induced by the inclusion

$$
s_{i}: k_{*} \backslash\{i\} \subset k_{*} .
$$

\footnotetext{
${ }^{6}$ The name stems from the fact that elements of $\Omega C_{N}$ can be understood as linear combinations of configurations of beads of $N$ colors arranged on a string.

${ }^{7}$ The representations $U_{N}^{I, I I}$ considered in Subsection 2.2 correspond to $U_{(N)}^{I, I I}$ in the new notation.
} 
The action of $\operatorname{End}\left(F_{n}\right)$ on $C H^{\vee_{n} S^{1}}\left(L_{*}\right)$ is defined analogously as that on $C H^{\vee_{n} S^{1}}\left(M \otimes C^{\otimes \bullet}\right)=M \otimes(\Omega C)^{\otimes n}$, see (9) 8 Notice that the coproduct on $\Omega C$ is the sum of coshuffles, and the product is just concatenation. Let $\gamma$ lie in the $\left(k_{1}, \ldots, k_{n}\right)$ component of (11), and $\Psi \in \operatorname{End}\left(F_{n}\right)$ is such that $x_{j}$ appears in total $r_{j}$ times in $\Psi\left(x_{1}\right), \Psi\left(x_{2}\right), \ldots, \Psi\left(x_{n}\right)$. One has that $\Psi^{*}(\gamma)$ is the sum of $r_{1}^{k_{1}} \cdot r_{2}^{k_{2}} \cdot \ldots \cdot r_{n}^{k_{n}}$ elements each of which is obtained from $\gamma$ by some permutation of its inputs. More concretely, $\Psi$ defines a map $\vee_{n} S^{1} \rightarrow \vee_{n} S^{1}$ such that any point on the $i$-th circle has exactly $r_{i}$ preimages. We put $k_{1}$ points on the first circle in the target wedge, $k_{2}$ on the second, ..., $k_{n}$ on the last one. These points correspond to the inputs of $\gamma$. For every point in the target we choose a preimage point (thus for the $i$-th circle there are $r_{i}^{k_{i}}$ choices making the total of $\prod_{i=1}^{n} r_{i}^{k_{i}}$ choices). For every such choice we get a collection of points on the source wedge, which contributes a summand in $\Psi^{*}(\gamma)$, that has to be taken with the sign of permutation of inputs of $\gamma$.

Consider examples similar to those given in Example 1.1

(a) $n=1 ; \Psi\left(x_{1}\right)=x_{1}^{2}$. In this case,

$$
\Psi^{*}\left(\gamma\left(x_{11}, \ldots, x_{1 k_{1}}\right)\right)=\sum_{i=0}^{k_{1}} \sum_{\sigma \in \operatorname{Sh}\left(i, k_{1}-i\right)}(-1)^{\sigma} \gamma\left(\sigma\left(x_{11}, \ldots, x_{1 k_{1}}\right)\right)
$$

Here and below $S h(i, j)$ denotes the set of shuffles of an $i$-elements set with a $j$-elements set.

(b) $n=1, \Psi\left(x_{1}\right)=x_{1}^{-1}$. In this case

$$
\Psi^{*}\left(\gamma\left(x_{11}, \ldots, x_{1 k_{1}}\right)\right)=(-1)^{\frac{k_{1}\left(k_{1}-1\right)}{2}} \gamma\left(x_{1 k_{1}}, \ldots, x_{11}\right) .
$$

(c) $n=2 ; \Psi\left(x_{1}\right)=x_{1} x_{2}, \Psi\left(x_{2}\right)=x_{2}$ :

$$
\Psi^{*}\left(\gamma\left(x_{11}, \ldots, x_{1 k_{1}}, x_{21}, \ldots, x_{2 k_{2}}\right)\right)=\sum_{i=0}^{k_{2}} \sum_{\sigma \in \operatorname{Sh}\left(i, k_{2}-i\right)}(-1)^{\sigma} \gamma\left(x_{11}, \ldots, x_{1 k_{1}}, \sigma\left(x_{1 k_{1}+1}, \ldots, x_{1 k_{1}+i}, x_{21}, \ldots, x_{2 k_{2}-i}\right)\right) .
$$

Proposition 3.1. The action of $\operatorname{End}\left(F_{n}\right)$ on $\mathrm{CH}^{\vee_{n} S^{1}}\left(L_{*}\right)$ defined above coincides in the homology with the topological action.

Idea of the proof. One can check that for all elements $\Psi \in \operatorname{End}\left(F_{n}\right)$ their action $\Psi^{*}$ on $C H^{\vee_{n} S^{1}}\left(L_{*}\right)$ can be decomposed into a composition of maps induced by multisimplicial maps, Eilenberg-Zilber maps (10), and some natural chain homotopy inverses to those maps.

This proposition is a partial case of Theorem 4. That's why we choose not to give a detailed proof of it, but only mention that there is a proof which goes through a careful study of multi-simplical maps. (This argument is similar to the explicit identification of the surface product studied in [15].) Indeed, Theorem 4 among other things states that the complexes $C H^{\vee_{n} S^{1}}\left(L_{*}\right)$ are identical to $\mathrm{CH}^{\vee_{n} S^{1}}\left(L_{*}\right)$, where the latter ones are constructed using the definition of the Hochschild-Pirashvili homology in terms of derived maps of right $\Gamma$ modules. Moreover, Remark 5.4 asserts that the induced action of $\operatorname{End}\left(F_{n}\right)$ on $\mathrm{CH}^{\vee_{n} S^{1}}\left(L_{*}\right)$ is identical to the one on $C H^{\vee_{n} S^{1}}\left(L_{*}\right)$ defined in this section. We will also see in Subsection 5.1 that the reason that the $\operatorname{End}\left(F_{n}\right)$ action on $H H^{\vee_{n} S^{1}}\left(L_{*}\right)$ can be lifted on the level of chains is the coformality of the induced $\operatorname{End}\left(F_{n}\right)$ action on the $\Omega$-module $C_{*}\left(\left(\vee_{n} S^{1}\right)^{\wedge} \bullet\right)$

Proof of Theorem 2. At this point we only need to explain what is the Hodge splitting in $C H^{\vee_{n} S^{1}}\left(L_{*}\right)$, why it is preserved by the $\operatorname{End}\left(F_{n}\right)$ action as a filtration, and why on the associated graded complex $\operatorname{grCH} H_{n}^{\mathrm{V}^{1}}\left(L_{*}\right)$ this action factors through $\operatorname{End}\left(\mathbb{Z}^{n}\right)$.

In case $n=1$, i.e. for the usual Hochschild complex $C H^{S^{1}}\left(L_{*}\right)$, the Hodge splitting is obtained by noticing that the action of $\operatorname{End}\left(F_{1}\right)=(\mathbb{Z}, *)$ splits this complex into a direct product of spaces numbered by non-negative integers, such that on the $m$-th component $r \in(\mathbb{Z}, *)$ acts as multiplication by $r^{m}$ [13, 21]. The projection on the $m$-th component is called $m$-th Euler idempotent $e_{m}$. Notice that each component $N L_{*}(\ell)[\ell]$ of the complex

$$
C H^{S^{1}}\left(L_{*}\right)=\operatorname{Tot}\left(L_{*} \circ S_{\bullet}^{1}\right)=\left(\prod_{\ell \geq 0} N L_{*}(\ell)[\ell], d\right)
$$

is acted on by $\mathbb{S}_{\ell}$ and thus by the group algebra $\mathbb{Q}\left[\mathbb{S}_{\ell}\right]$. The Euler idempotent $e_{m}(\ell)$ is obtained via this action and is in fact an element of $\mathbb{Q}\left[\mathbb{S}_{\ell}\right]$. To give a bit more insight, one has an isomorphism of symmetric sequences:

$$
\text { Com } \circ \text { Lie } \stackrel{\simeq}{\longrightarrow} \text { Assoc, }
$$

\footnotetext{
${ }^{8}$ Recall that we assume that $C$ is simply connected. If we only assume that $C$ is connected, than the complex $C H^{\vee_{n} S^{1}}\left(M \otimes C^{\otimes \bullet}\right)$ is $M \hat{\otimes}(\Omega C)^{\otimes n}$, where instead of the cobar complex we take the completed cobar and instead of tensor product the completed tensor product.

${ }^{9}$ By this we mean that every induced map of the action is coformal, see Definition 5.1 and Proposition 5.2
} 
induced by the Poincaré-Birkhoff-Witt map. The image of $e_{m}(\ell)$ is exactly

$$
[\operatorname{Com}(m) \circ \operatorname{Lie}](\ell) \subset \operatorname{Assoc}(\ell)=\mathbb{Q}\left[S_{\ell}\right] .
$$

When $n \geq 2$, to obtain a similar splitting in Hochschild-Pirashvili homology one can use the action of the monoid $(\mathbb{Z}, *)^{\times n} \subset \operatorname{End}\left(F_{n}\right)$ consisting of the homotopy classes of maps $\vee_{n} S^{1} \rightarrow \vee_{n} S^{1}$ sending each circle into itself. The complex $C H^{\vee} S^{1}\left(L_{*}\right)$ splits into a direct product of spaces numbered by $n$-tuples $\left(m_{1}, \ldots, m_{n}\right)$ of non-negative integers. Element $\left(r_{1}, \ldots, r_{n}\right) \in(\mathbb{Z}, *)^{\times n}$ acts on the $\left(m_{1}, \ldots, m_{n}\right)$ component of the Hodge splitting as multiplication by $r_{1}^{m_{1}} \cdot \ldots \cdot r_{n}^{m_{n}}$. Each $\left(\ell_{1}, \ldots, \ell_{n}\right)$ component $N L_{*}\left(\ell_{1}+\ldots+\ell_{n}\right)$ of $\operatorname{Tot}(L_{*} \circ\left(\vee_{n} S^{1}\right) \underbrace{\bullet \ldots}_{n})$ is acted on by $\mathbb{S}_{\ell_{1}} \times \ldots \times \mathbb{S}_{\ell_{n}}$. The projection onto the $\left(m_{1}, \ldots, m_{n}\right)$ Hodge component is given by $e_{m_{1}}\left(\ell_{1}\right) \otimes \ldots \otimes^{n} e_{m_{n}}\left(\ell_{n}\right)$. We define the total Hodge degree as $m=m_{1}+\ldots+m_{n}$. One can see that the action of $\operatorname{End}\left(F_{n}\right)$ preserves it as a filtration.

To see that the $\operatorname{End}\left(F_{n}\right)$ action on $\operatorname{grCH}^{\mathrm{V}_{n} S^{1}}\left(L_{*}\right)$ factors through $\mathrm{GL}(n, \mathbb{Z})$, see equations (22), (23), and Remark 4.7, which describe $g r C H^{\vee_{n} S^{1}}\left(L_{*}\right)$ in terms of $H^{1}\left(\vee_{n} S^{1}\right)$.

\section{Hochschild-Pirashvili homology on suspensions. Proof of Theorem 3}

4.1. Complexes $g r \mathrm{CH}^{\Sigma Y_{*}}\left(L_{*}\right)$. In this subsection we describe complexes computing higher Hochschild homology on suspensions $H H^{\Sigma Y_{*}}\left(L_{*}\right)$. These complexes depend only on $\tilde{H}_{*}\left(Y_{*}\right)$ and as we will later see in Subsection 5.1 they can be naturally identified with the associated graded of $\mathrm{CH}^{\Sigma Y_{*}}\left(L_{*}\right)$.

One of the two reasons for the Hodge splitting in the higher Hochschild homology (on a suspension) is the formality of the $\Gamma$-module $C_{*}\left(X_{*}^{*}\right)$ in case $X_{*}=\Sigma Y_{*}$. Recall that a $\Gamma$-module is said formal if it is quasi-isomorphic via a zigzag of quasi-isomorphisms to its homology $\Gamma$-module. Similarly, a map between $\Gamma$-modules is formal if this map is quasi-isomorphic via a zigzag of quasi-isomorphisms of $\Gamma$-modules maps to the induced map in their homology.

Lemma 4.1. If a pointed space $X_{*}$ is of finite type and is rationally formal, then the right $\Gamma$ module $C_{*}\left(X_{*}^{*}\right)$ is also rationally formal. If a pointed map $X_{*} \rightarrow Y_{*}$ between spaces of finite type is rationally formal, then the induced map of $\Gamma$ modules $C_{*}\left(X_{*}^{*}\right) \rightarrow C_{*}\left(Y_{*}^{*}\right)$ is also formal.

Proof. By formality of a space we understand formality of its Sullivan algebra $A_{X_{*}}$ as augmented algebra and similarly for a map between spaces. We show explicitly the first statement. The second one follows from functoriality of the construction. One has a quasi-isomorphism of $\Gamma$-modules:

$$
C_{*}\left(X_{*}^{\bullet}\right) \simeq\left(A_{X_{*}^{*}}\right)^{\vee} \simeq\left(\mathbb{Q} \otimes A_{X_{*}}^{\otimes \bullet}\right)^{\vee} \simeq\left(\mathbb{Q} \otimes H^{*}\left(X_{*}\right)^{\otimes \bullet}\right)^{\vee} \simeq H_{*}\left(X_{*}^{\bullet}\right) .
$$

Lemma 4.2. Any suspension of a space of finite type is rationally formal and, moreover, any suspension of a map between spaces of finite type is rationally formal.

Recall that a map of pointed spaces is formal if the induced map of Sullivan augmented algebras is formal, i.e., quasi-isomorphic to the map of rational cohomology algebras (in the category of augmented algebras). In particular it implies that each space is formal.

Proof. Let $Y_{*}$ be a space of finite type and let us show that $\Sigma Y_{*}$ is formal. The argument for a map between suspensions is similar. In case $Y_{*}$ is connected, its suspension $\Sigma Y_{*}$ is simply connected. It is also a co- $H$-space, therefore it is coformal and its Quillen model is a free Lie algebra generated by $\tilde{H}_{*}\left(Y_{*}\right)$ with zero differential. The Koszul dual commutative algebra is generated by $\tilde{H}^{*}\left(\Sigma Y_{*}\right)$ with all products of generators being zero.

In case $Y_{*}=\bigsqcup_{i=1}^{k} Y_{i}$ is a disjoint union of $k$ components, then $\Sigma Y_{*}=\left(\bigvee_{k-1} S^{1}\right) \vee\left(\bigvee_{i=1}^{k} \Sigma Y_{i}\right)$. And the wedge of formal spaces is formal.

Notice that from these two lemmas it follows that if $X_{*}$ is a suspension of finite type, then $C_{*}\left(X_{*}^{*}\right)$ is a formal $\Gamma$-module and that the same is true for a suspension of a map between spaces of finite type. Proposition 4.4 below implies that the finiteness condition can be released.

Let $\Omega$ be the category of finite sets with morphisms all surjective maps. In [27] Pirashvili defines an equivalence of categories

$$
c r: \bmod -\Gamma \rightarrow \bmod -\Omega \text {. }
$$

On objects

$$
\operatorname{cr} L_{*}(k)=L_{*}\left(k_{*}\right) /+_{i=1}^{k} \operatorname{Im} r_{i}^{*},
$$


where $r_{i}^{*}: L_{*}\left(k_{*} \backslash\{i\}\right) \rightarrow L_{*}\left(k_{*}\right)$ is induced by the map $r_{i}: k_{*} \rightarrow k_{*} \backslash\{i\}$ :

$$
r_{i}(j)= \begin{cases}j, & j \neq i \\ *, & j=i\end{cases}
$$

On morphisms $\operatorname{cr} L_{*}$ is obtained as restriction with respect to the inclusion $i: \Omega \rightarrow \Gamma$ that adds the basepoint to any set: $i(k)=k_{*}$. Recall (12). The space $\operatorname{cr} L_{*}(k)$ is isomorphic to $N L_{*}(k)$ via the obvious composition

$$
q: N L_{*}(k) \hookrightarrow L_{*}\left(k_{*}\right) \rightarrow \operatorname{cr} L_{*}(k) .
$$

One can show that $q$ is an isomorphism using the map $\prod_{i=1}^{k}\left(1-r_{i}^{*} s_{i}^{*}\right)$ that projects $L_{*}\left(k_{*}\right)$ onto $N L_{*}(k)$. (Notice that $r_{i}^{*} s_{i}^{*}, i=1 \ldots k$, are pairwise commuting projectors as well as $\left(1-r_{i}^{*} s_{i}^{*}\right), i=1 \ldots k$.) For the complexes that we consider below it is sometimes convenient to use $N L_{*}(\bullet)$ instead of $\operatorname{cr} L_{*}(\bullet)$.

Let us describe the induced $\Omega$-module structure on $N L_{*}(\bullet)$. The symmetric group action as part of $\Omega$ structure on $N L_{*}(\bullet)$ is the usual one. Denote by $m_{i}:(k+1) \rightarrow k$ the surjection

$$
m_{i}(j)= \begin{cases}j, & 1 \leq j \leq i \\ j-1, & i+1 \leq j \leq k\end{cases}
$$

Abusing notation we denote by $m_{i}:(k+1)_{*} \rightarrow k_{*}$ the same map extended as $m_{i}: * \mapsto *$. For $\gamma \in \operatorname{cr} L_{*}(k)$, one has

$$
q^{-1}\left(m_{i}^{*}(\gamma)\right)=\left(1-r_{i}^{*} s_{i}^{*}-r_{i+1}^{*} s_{i+1}^{*}\right) m_{i}^{*}\left(q^{-1}(\gamma)\right) .
$$

One can write this formula slightly differently. Recall that the structure of a right $\Omega$-module is equivalent to the structure of a right module over the commutative non-unital operad $\mathrm{Com}_{+}$, while the structure of a right $\Gamma$ module is equivalent to the structure of an infinitesimal bimodule over the commutative unital operad Com, see [2, Proposition 4.9] or [32, Lemma 4.3]. In this terms, equation (15) is written as

$$
\begin{aligned}
q^{-1}\left(\gamma\left(x_{1}, \ldots, x_{i} \cdot x_{i+1}, \ldots, x_{k+1}\right)\right)=q^{-1}(\gamma) & \left(x_{1}, \ldots, x_{i} \cdot x_{i+1}, \ldots, x_{k+1}\right) \\
& \quad-x_{i} \cdot q^{-1}(\gamma)\left(x_{1} \ldots \hat{x}_{i} \ldots x_{k+1}\right)-x_{i+1} \cdot q^{-1}(\gamma)\left(x_{1} \ldots \hat{x}_{i+1} \ldots x_{k+1}\right) .
\end{aligned}
$$

The two last summands in (15) and (16) are correction terms necessary to make the right-hand side normalized.

The higher Hochschild homology over a pointed space $X_{*}$ is computed as the space of homotopy maps of $\Gamma$-modules

$$
H H^{X_{*}}\left(L_{*}\right)=H_{*}\left(\operatorname{hRmod}_{\Gamma}\left(C_{*}\left(X_{*}^{*}\right), L_{*}\right)\right) \text {. }
$$

For any pointed space $X_{*}$, the cross-effect of the $\Gamma$-module $C_{*}\left(X_{*}^{*}\right)$ is equivalent to

$$
\operatorname{cr} C_{*}\left(X_{*}^{\bullet}\right) \simeq \tilde{C}_{*}\left(X_{*}^{\wedge}\right),
$$

see [1], where the $\Omega$-module structure on $\tilde{C}_{*}\left(X_{*}^{\wedge \bullet}\right)$ is induced by the diagonal maps. For any surjection $p: k \rightarrow \ell$, one gets a map $X_{*}^{\wedge \ell} \rightarrow X_{*}^{\wedge k}$ defined as

$$
\left(x_{1}, \ldots x_{\ell}\right) \mapsto\left(x_{p^{-1}(1)}, \ldots, x_{p^{-1}(k)}\right) .
$$

It follows that the Hochschild-Pirashvili homology can also be described as

$$
H H^{X_{*}}\left(L_{*}\right)=H_{*}\left(\mathrm{hRmod}_{\Omega}\left(\tilde{C}_{*}\left(X_{*}^{\wedge \bullet}\right), \operatorname{cr} L_{*}\right)\right) \text {. }
$$

Definition 4.3. We say that a right $\Omega$ module $M$ has a trivial $\Omega$ action if for any strict surjection $p: k \rightarrow \ell$ the induced map $M(\ell) \rightarrow M(k)$ is the zero map.

Proposition 4.4. For any pointed suspension $\Sigma Y_{*}$, the $\Omega$ module $\tilde{C}_{*}\left(\left(\Sigma Y_{*}\right)^{\wedge \bullet}\right)$ is formal. For any pointed map $g: Y_{*} \rightarrow Z_{*}$, the induced map of $\Omega$ modules $(\Sigma g)_{*}: \tilde{C}_{*}\left(\left(\Sigma Y_{*}\right)^{\wedge \bullet}\right) \rightarrow \tilde{C}_{*}\left(\left(\Sigma Z_{*}\right)^{\wedge}\right)$ is also formal.

Proof. For the proof we will need that the $\Omega$ module $\tilde{C}_{*}\left(\left(S^{1}\right)^{\wedge}\right)$ is formal and has the trivial $\Omega$ action in homology. The first statement follows from the fact that the $\Gamma$ module $C_{*}\left(\left(S^{1}\right)^{\bullet}\right)$ is formal (by Lemmas 4.1 and 4.2) and thus is so its cross-effect $\operatorname{cr} C_{*}\left(\left(S^{1}\right)^{\bullet}\right) \simeq \tilde{C}_{*}\left(\left(S^{1}\right)^{\wedge}\right)$. The second statement is straightforward as any diagonal map $S^{\ell} \rightarrow S^{k}$ for $k>\ell$ induces the zero map in reduced homology.

The following sequence of quasi-isomorphisms of $\Omega$ modules proves the formality of $\tilde{C}_{*}\left(\left(\Sigma Y_{*}\right)^{\wedge}\right)[$

$$
\begin{aligned}
& \tilde{C}_{*}\left(\left(\Sigma Y_{*}\right)^{\wedge \bullet}\right) \simeq \tilde{C}_{*}\left(\left(S^{1}\right)^{\wedge \bullet}\right) \otimes \tilde{C}_{*}\left(Y_{*}^{\wedge \bullet}\right) \simeq \tilde{H}_{*}\left(\left(S^{1}\right)^{\wedge \bullet}\right) \otimes \tilde{C}_{*}\left(Y_{*}^{\wedge \bullet}\right) \simeq \\
& \simeq \tilde{H}_{*}\left(\left(S^{1}\right)^{\wedge \bullet}\right) \otimes \tilde{C}_{*}\left(Y_{*}\right)^{\otimes \bullet} \simeq \tilde{H}_{*}\left(\left(S^{1}\right)^{\wedge \bullet}\right) \otimes \tilde{H}_{*}\left(Y_{*}\right)^{\otimes \bullet} .
\end{aligned}
$$

By the tensor product above we understand an objectwise tensor product of right $\Omega$ modules. The second quasiisomorphism uses the formality of $\tilde{C}_{*}\left(\left(S^{1}\right)^{\wedge}\right)$. Notice that all the terms in this zigzag starting from the third one have the trivial $\Omega$ action. Notice also that all the quasi-isomorphisms are functorial in $Y_{*}$ except the last one, which

\footnotetext{
${ }^{10}$ This simple argument was provided to us by G. Arone.
} 
uses a choice of a quasi-isomorphism $\tilde{H}_{*} Y_{*} \rightarrow \tilde{C}_{*} Y_{*}$. On the other hand, any morphism of complexes (in our case $\tilde{C}_{*} Y_{*} \rightarrow \tilde{C}_{*} Z_{*}$ ) is formal (i.e., is quasi-isomorphic to the induced map $\tilde{H}_{*} Y \rightarrow \tilde{H}_{*} Z$ ). This proves the formality of the induced map of $\Omega$ modules.

Remark 4.5. It follows from $(19)$ that for any suspension $\Sigma Y_{*}$, the right $\Omega$ module $\tilde{C}_{*}\left(\left(\Sigma Y_{*}\right)^{\wedge}\right)$ has the trivial $\Omega$ action in homology.

This property is in fact the second of the two reasons for the Hodge splitting. (The first one is the formality.) Indeed, as a consequence, the $\Omega$-module $\tilde{H}_{*}\left(\left(\Sigma Y_{*}\right)^{\wedge}\right)$ splits into a direct sum of $\Omega$-modules:

$$
\operatorname{cr} \tilde{C}_{*}\left(\left(\Sigma Y_{*}\right)^{\wedge \bullet}\right) \simeq \tilde{H}_{*}\left(\left(\Sigma Y_{*}\right)^{\wedge \bullet}\right) \simeq \bigoplus_{m \geq 0} \tilde{H}_{*}\left(\Sigma Y_{*}\right)^{\otimes m},
$$

where $\tilde{H}_{*}\left(\Sigma Y_{*}\right)^{\otimes m}$ denotes the $\Omega$-module which is $\tilde{H}_{*}\left(\Sigma Y_{*}\right)^{\otimes m}$ in arity $m$ and 0 in all others. Thus we get

$$
H H^{\Sigma Y_{*}}\left(L_{*}\right) \simeq \prod_{m \geq 0} H\left(\operatorname{hRmod}_{\Omega}\left(\tilde{H}_{*}\left(\Sigma Y_{*}\right)^{\otimes m}, \operatorname{cr} L_{*}\right)\right) .
$$

As a corollary we see that the functor $H H^{(-)}\left(L_{*}\right)$ factors through the reduced homology functor $\tilde{H}_{*}:$ Top $p_{*} \rightarrow$ $g$ Vect when restricted on $\Sigma\left(T o p_{*}\right)$. The splitting by $m$ in (21) is exactly the Hodge splitting.

Now we want to make more explicit the right-hand side of (21). Recall that the right $\Omega$-module is the same as the right $\mathrm{Com}_{+}$-module. Applying the Koszul duality between the Lie and Com $\mathrm{C}_{+}$operads, the cofibrant replace-

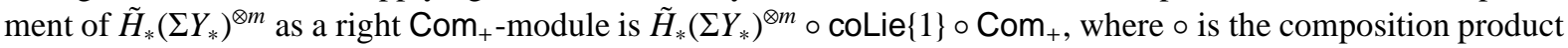
of symmetric sequences; coLie is the Lie cooperad; $\{1\}$ denotes operadic suspension [11, 2, 31]. The differential in it is obtained by taking off one cobracket from the coLie $\{1\}$ factor and by making it act from the left on the Com $_{+}$part as a product $x_{1} \cdot x_{2}$, see [2, Section 5]. For a general right Com + -module $M$, there is another term of the differential on its cofibrant replacement $M \circ \operatorname{coLie}\{1\} \circ \mathrm{Com}_{+}$, which takes off one cobracket from the coLie $\{1\}$ part and makes it act from the right on $M$ also as a product $x_{1} \cdot x_{2}$. But in our case this action is trivial, so only the first part of the differential is present. The product over $m \geq 0$ of the complexes below computes $H H^{\Sigma Y_{*}}\left(L_{*}\right)$ :

$$
\begin{aligned}
& \operatorname{Rmod}_{\mathrm{Com}_{+}}\left(\tilde{H}_{*}\left(\Sigma Y_{*}\right)^{\otimes m} \circ \operatorname{coLie}\{1\} \circ \operatorname{Com}_{+}, \operatorname{cr} L_{*}\right)=\left(\operatorname{Hom}\left(\tilde{H}_{*}\left(\Sigma Y_{*}\right)^{\otimes m} \circ \operatorname{coLie}\{1\}, \operatorname{cr} L_{*}\right), d\right)= \\
& \operatorname{Hom}_{m}\left(\tilde{H}_{*}\left(Y_{*}\right)^{\otimes m},\left(\prod_{\ell \geq m} \bigoplus_{\ell_{1}+\ldots+\ell_{m}=\ell}\left(\operatorname{Lie}\left(\ell_{1}\right) \otimes \ldots \otimes \operatorname{Lie}\left(\ell_{m}\right) \otimes_{\ell_{1} \times \ldots \times \ell_{m}}\left(\operatorname{sign} \otimes \operatorname{cr} L_{*}(\ell)\right)\right)[\ell], d\right)\right),
\end{aligned}
$$

which assuming the finiteness condition on the homology of $Y_{*}$ can also be written as

$$
\tilde{H}^{*}\left(Y_{*}\right)^{\otimes m} \hat{\otimes}{ }_{m}\left(\prod_{\ell \geq m} \bigoplus_{\ell_{1}+\ldots+\ell_{m}=\ell}\left(\operatorname{Lie}\left(\ell_{1}\right) \otimes \ldots \otimes \operatorname{Lie}\left(\ell_{m}\right) \otimes_{\ell_{1} \times \ldots \times \ell_{m}}\left(\operatorname{sign} \otimes \operatorname{cr} L_{*}(\ell)\right)\right)[\ell], d\right) .
$$

Here sign denotes the sign representation of $\mathbb{S}_{\ell}$; the reduced cohomology of $Y_{*}$ is viewed as a negatively graded vector space. The differential in this complex is the sum of simultaneous insertions of $\left[x_{1}, x_{2}\right]$ in one of the inputs of $\operatorname{Lie}\left(\ell_{i}\right)$ for some $i$, and right action by $x_{1} \cdot x_{2}$ on the corresponding input of $\operatorname{cr} L_{*}(\ell)$. Beware that if we replace $\operatorname{cr} L_{*}(\ell)$ by $N L_{*}(\ell)$ additional summands in the differential appear due to the last two terms in (15)-(16).

Remark 4.6. In case $Y_{*}$ is of finite type, and $L_{*}=M \otimes C^{\otimes \bullet}$, the obtained complex computing $H H^{\Sigma Y_{*}}(C, M)$ is

$$
M \hat{\otimes} S\left(\tilde{H}^{*}\left(Y_{*}\right) \hat{\otimes} \mathcal{L}(C)\right),
$$

where the cohomology $\tilde{H}^{*}\left(Y_{*}\right)$ is non-positively graded; $\mathcal{L}(C)$ is the Harrison complex of $C$. The symmetric power and tensor products are the completed ones. The differential

$$
d=d_{M}+d_{C}+\delta
$$

where $d_{M}$ and $d_{C}$ are induced by the differential on $M$ and $\mathcal{L}(C)$, and $\delta(m \otimes x)=m^{\prime} \otimes\left[m^{\prime \prime}, x\right]$. The part $\delta$ in the differential appears due to the last two summands in (15)-16. 11

Remark 4.7. For $Y_{*}=\vee_{n} S^{0}$ and any $L_{*}$, the obtained complex is identical to $\operatorname{gr} C H_{*}^{\vee_{n} S^{1}}\left(L_{*}\right)$ considered in Section 3 In case $L_{*}=M \otimes C^{\otimes \bullet}$ it follows from Proposition 1.3 and Remark 4.6. For a general $L_{*}$ one can construct this isomorphism analogously. The idea is that elements of $\operatorname{Lie}\left(\ell_{i}\right)$ in 23 should be viewed as linear combinations of permutations in $\mathbb{S}_{\ell_{i}}$, which tells us in which order the elements should be put on the corresponding circle.

\footnotetext{
${ }^{11}$ To recall $C$ is simply connected. If $C$ is not simply connected, the Harrison complex $\mathcal{L}(C)$ should be replaced by the completed Harrison complex $\hat{\mathcal{L}}(C)$.
} 
4.2. Hodge filtration. Proof of Theorem 3. We define a functorial filtration on the space of homotopy maps of right $\Omega$-modules, which induces the desired filtration on $H H^{X_{*}}\left(L_{*}\right)$ functorial in $X_{*}$ and $L_{*}$. For a right $\Omega$-module $K$ define its $m$-th truncation $t r_{m} K$ as

$$
\operatorname{tr}_{m}(K)(\ell)= \begin{cases}K(\ell), & \ell \leq m ; \\ 0, & \ell>m .\end{cases}
$$

This symmetric sequence has an obvious $\Omega$-module structure, such that the projection $K \rightarrow t r_{m} K$ is an $\Omega$-modules map. This morphism for any $\Omega$-module $L$ induces a map of complexes

$$
\operatorname{hRmod}_{\Omega}\left(\operatorname{tr}_{m} K, L\right) \rightarrow \operatorname{hRmod}_{\Omega}(K, L) .
$$

Its image in homology is what we call the $m$-th term of the Hodge filtration in $H\left(\operatorname{hRmod}_{\Omega}(K, L)\right)$.

For $K=\tilde{C}_{*}\left(\left(\Sigma Y_{*}\right)^{\wedge \bullet}\right) \simeq \tilde{H}_{*}\left(\Sigma Y_{*}\right)^{\otimes \bullet}$, the cofiltration $t r_{\bullet}$ splits. For any pointed map of suspensions $\Sigma Y_{*} \rightarrow \Sigma Z_{*}$, the induced map

$$
\operatorname{grHH} H^{\Sigma Z_{*}}\left(L_{*}\right) \rightarrow \operatorname{grHH} H^{\Sigma Y_{*}}\left(L_{*}\right)
$$

can be recovered from the map of the layers of $t r_{\bullet}$ (and thus from the map in homology $\tilde{H}_{*} \Sigma Y_{*} \rightarrow \tilde{H}_{*} \Sigma Z_{*}$ ) by the spectral sequence argument.

4.3. Hodge filtration versus cardinality cofiltration. Denote by $C \mathcal{H}^{X_{*}}\left(L_{*}\right)$ the higher Hochschild complex

$$
C \mathcal{H}^{X_{*}}\left(L_{*}\right):=\operatorname{hRmod}_{\Omega}\left(\tilde{C}_{*}\left(X_{*}^{\wedge \bullet}\right), \operatorname{cr} L_{*}\right) .
$$

The Hodge filtration

$$
F_{0} C \mathcal{H}^{X_{*}}\left(L_{*}\right) \rightarrow F_{1} C \mathcal{H}^{X_{*}}\left(L_{*}\right) \rightarrow F_{2} C \mathcal{H}^{X_{*}}\left(L_{*}\right) \rightarrow \ldots
$$

should not be confused with the more widely used cardinality or rank cofiltration (depending on the context it can also be called Goodwillie-Weiss tower) [3, 18, 35]:

$$
T_{0} C \mathcal{H}^{X_{*}}\left(L_{*}\right) \leftarrow T_{1} C \mathcal{H}^{X_{*}}\left(L_{*}\right) \leftarrow T_{2} C \mathcal{H}^{X_{*}}\left(L_{*}\right) \leftarrow \ldots
$$

We have seen in the previous subsection that

$$
F_{m} C \mathcal{H}^{X_{*}}\left(L_{*}\right) \simeq \operatorname{hRmod}_{\Omega}\left(t r_{m} \tilde{C}_{*}\left(X_{*}^{\wedge \bullet}\right), \operatorname{cr} L_{*}\right) .
$$

Proposition 4.8. The $n$-th term of the cardinality cofiltration is

$$
T_{m} C \mathcal{H}^{X_{*}}\left(L_{*}\right) \simeq \operatorname{hRmod}_{\Omega}\left(\tilde{C}_{*}\left(X_{*}^{\wedge \bullet}\right), \operatorname{tr}{ }_{m} c r L_{*}\right) .
$$

Proof. Denote by $\Gamma_{m}$ and $\Omega_{m}$ the full subcategories of $\Gamma$, respectively $\Omega$, consisting of objects of cardinal $\leq m+1$, respectively $\leq m$. One has obvious restriction functors

$$
\left.(-)\right|_{\leq m}: \bmod -\Gamma \rightarrow \bmod -\Gamma_{m} ;\left.\quad(-)\right|_{\leq m}: \bmod -\Omega \rightarrow \bmod -\Omega_{m} .
$$

By definition

$$
T_{m} C \mathcal{H}^{X_{*}}\left(L_{*}\right) \simeq \operatorname{hRmod}_{\Gamma_{m}}\left(\left.C_{*}\left(X_{*}^{\bullet}\right)\right|_{\leq m},\left.L_{*}\right|_{\leq m}\right) .
$$

The cross-effect functor

$$
c r: \bmod -\Gamma_{m} \rightarrow \bmod -\Omega_{m}
$$

defined by (13) is also an equivalence in the truncated case.

For a right $\Omega_{m}$ module $K$, denote by $\operatorname{triv}_{m}(K)$ the $\Omega$ module extended trivially on sets of cardinal $>m$ :

$$
\operatorname{triv}_{m}(K)(\ell)= \begin{cases}K(\ell), & \ell \leq m ; \\ 0, & \ell>m .\end{cases}
$$

One has a Quillen adjunction

$$
\left.(-)\right|_{\leq m}: \bmod -\Omega \rightleftarrows \bmod -\Omega_{m}: \text { triv } v_{m} .
$$

Notice that $\operatorname{rriv}_{m} \circ(-)_{\leq m}=t r_{m}$. As a consequence we get

$$
T_{m} C \mathcal{H}^{X_{*}}\left(L_{*}\right) \simeq \operatorname{hRmod}_{\Omega_{m}}\left(\left.\tilde{C}_{*}\left(X_{*}^{\wedge \bullet}\right)\right|_{\leq m},\left.\operatorname{cr} L_{*}\right|_{\leq m}\right) \simeq \operatorname{hRmod}_{\Omega}\left(\tilde{C}_{*}\left(X_{*}^{\wedge \bullet}\right), \operatorname{tr} r_{m} c r L_{*}\right) .
$$


Finally, let us compare the $T_{m}$ and $F_{m}$ terms in case of a suspension to make sure that they are different.

$$
\begin{gathered}
F_{m} \mathrm{CH}^{\Sigma Y_{*}}\left(L_{*}\right)=\prod_{i=0}^{m} \operatorname{hRmod}_{\Omega}\left(\tilde{H}_{*}\left(\Sigma Y_{*}\right)^{\otimes i}, \operatorname{cr} L_{*}\right)=\prod_{i=0}^{m}\left(\prod_{j=i}^{+\infty} \operatorname{Hom}_{j}\left(\left(\tilde{H}_{*}\left(\Sigma Y_{*}\right)^{\otimes i} \circ \operatorname{coLie}\{1\}\right)(j), \operatorname{cr} L_{*}(j)\right), d\right) ; \\
T_{m} \mathrm{CH}^{\Sigma Y_{*}}\left(L_{*}\right)=\prod_{i=0}^{+\infty} \operatorname{hRmod}_{\Omega}\left(\tilde{H}_{*}\left(\Sigma Y_{*}\right)^{\otimes i}, \operatorname{tr} m \operatorname{cr} L_{*}\right)=\prod_{i=0}^{m}\left(\prod_{j=i}^{m} \operatorname{Hom}_{j}\left(\left(\tilde{H}_{*}\left(\Sigma Y_{*}\right)^{\otimes i} \circ \operatorname{coLie}\{1\}\right)(j), \operatorname{cr} L_{*}(j)\right), d\right) .
\end{gathered}
$$

One can see that the terms $F_{m}$ and $T_{m}$ are not the same.

Remark 4.9. The cardinality cofiltration induces a decreasing filtration in $\mathrm{CH}^{\Sigma Y_{*}}\left(L_{*}\right)$ : we define $F^{m} \mathrm{CH}^{\Sigma Y_{*}}\left(L_{*}\right)$ as the kernel of the projection $p_{m}: \mathrm{CH}^{\Sigma Y_{*}}\left(L_{*}\right) \rightarrow T_{m} \mathrm{CH}^{\Sigma Y_{*}}\left(L_{*}\right)$. Notice that $p_{m}$ restricted on $F_{m} \mathrm{CH}^{\Sigma Y_{*}}\left(L_{*}\right)$ is still surjective. As a consequence, one has that the Hodge filtration in the Hochschild-Pirashvili homology on a suspension is dense in the topology induced by this decreasing filtration.

Remark 4.10. The cardinality cofiltration in the higher Hochschild homology on suspensions, contrary to the Hodge filtration, does not split in general.

\section{Coformality of $C_{*}\left(\left(\Sigma Y_{*}\right)^{\wedge} \bullet\right)$. Proof of Theorem 4}

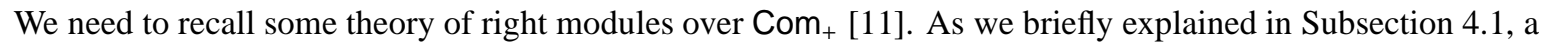
functorial cofibrant replacement of a right $\Omega$-module or equivalently a right $\mathrm{Com}_{+}$-module $M$ is $M \circ \operatorname{coLie}\{1\} \circ$ $\mathrm{Com}_{+}$. The sequence $M \circ$ coLie $\{1\}$ is the Koszul dual of $M$. Notice that it is naturally a right coLie $\{1\}$-comodule.

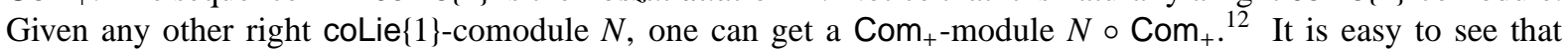
$N \circ \mathrm{Com}_{+}$is quasi-isomorphic to $M$ (as a Com + -module) if and only if $N$ is quasi-isomorphic to $M \circ \operatorname{coLie}\{1\}$ (as a coLie\{1\}-comodule). If this happens we say that $N$ is a Koszul dual of $M$ and $M$ is a Koszul dual of $N$.

This is part of a general homotopy theory of right modules [11]. For any right module $M$ over any doubly reduced operad $O$ in chain complexes $(O(0)=0, O(1)=\mathbb{Q})$, the bar construction $B(M, O, I)$ is a right comodule over the cooperad $B(I, O, I)$. By $I$ we mean the unit object in symmetric sequences

$$
I(k)= \begin{cases}\mathbb{Q}, & k=1 ; \\ 0, & k \neq 1 .\end{cases}
$$

In our case the operad $O=\mathrm{Com}_{+}$is Koszul and the bar complexes can be replaced by equivalent Koszul complexes [11].

It was shown by [1, Lemma 11.4], that for any pointed space $X_{*}$, the Koszul dual of $\tilde{C}_{*}\left(X_{*}^{\wedge \bullet}\right)$ is $\tilde{C}_{*}\left(X_{*}^{\wedge \bullet} / \Delta^{\bullet} X_{*}\right)$, where by $\Delta^{n} X_{*}$ we understand the fat diagonal in $X_{*}^{\wedge n}$. On homology the coLie $\{1\}$ coaction

$$
\circ_{i \sim j}: \tilde{H}_{*}\left(X_{*}^{\wedge n} / \Delta^{n} X_{*}\right) \rightarrow \tilde{H}_{*-1}\left(X_{*}^{\wedge n-1} / \Delta^{n-1} X_{*}\right) \otimes \operatorname{coLie}\{1\}(2)
$$

is induced by the connecting homomorphisms $\partial: H_{*}\left(X_{*}^{\wedge n}, \Delta^{n} X_{*}\right) \rightarrow H_{*-1}\left(\Delta^{n} X_{*}, \Delta_{i j}^{n} X_{*}\right)$ of the long exact sequence for the triples

$$
\left(X_{*}^{\wedge n}, \Delta^{n} X_{*}, \Delta_{i j}^{n} X_{*}\right)
$$

where $\Delta_{i j}^{n} X_{*}$ is the union of all diagonals except one: $x_{i}=x_{j}$. (One obviously has $\Delta^{n} X_{*} / \Delta_{i j}^{n} X_{*} \cong X_{*}^{\wedge n-1} / \Delta^{n-1} X_{*}$.)

Definition 5.1. We say that a right $\mathrm{Com}_{+}$-module is coformal if its Koszul dual coLie\{1\}-comodule is formal. A map of right $\mathrm{Com}_{+}-$modules is said coformal if the induced morphism of their Koszul duals is formal.

Proposition 5.2. For any pointed suspension $\Sigma Y_{*}$, the right $\mathrm{Com}_{+}-$module $\tilde{C}_{*}\left(\left(\Sigma Y_{*}\right)^{\wedge}\right)$ is coformal. For any pointed map of suspensions $f: \Sigma Y_{*} \rightarrow \Sigma Z_{*}$, the induced map of $\mathrm{Com}_{+}$-modules $f_{*}: \tilde{C}_{*}\left(\left(\Sigma Y_{*}\right)^{\wedge \bullet}\right) \rightarrow \tilde{C}_{*}\left(\left(\Sigma Z_{*}\right)^{\wedge \bullet}\right)$ is coformal.

Proof. According to Proposition 4.4 both Com - -modules $\tilde{C}_{*}\left(\left(\Sigma Y_{*}\right)^{\wedge} \bullet\right)$ and $\tilde{C}_{*}\left(\left(\Sigma Z_{*}\right)^{\wedge \bullet}\right)$ are formal. Their Koszul duals are $\tilde{H}_{*}\left(\Sigma Y_{*}\right)^{\otimes \bullet} \circ \operatorname{coLie}\{1\}$ and $\tilde{H}_{*}\left(\Sigma Z_{*}\right)^{\otimes \bullet} \circ \operatorname{coLie}\{1\}$, see Subsection 4.1, which are formal and cofree. On the other hand it is easy to see that any map between right coLie $\{1\}$-comodules whose homology is cofree, is formal.

Corollary 1. One has a natural isomorphism of right coLie $\{1\}$-comodules

$$
\tilde{H}_{*}\left(\left(\Sigma Y_{*}\right)^{\wedge \bullet} / \Delta^{\bullet} Y_{*}\right) \stackrel{\simeq}{\longrightarrow} \tilde{H}_{*}\left(\Sigma Y_{*}\right)^{\otimes \bullet} \circ \operatorname{coLie}\{1\},
$$

functorial over the category $\Sigma\left(\operatorname{Top}_{*}\right)$.

\footnotetext{
${ }^{12}$ The differential in $N \circ$ Com $_{+}$is the sum of two terms: the first one being induced by the differential on $N$, the second splits off one cobracket from $N$ and makes it act from the left as a product on $\mathrm{Com}_{+}$.
} 
One simply needs to apply the Koszul duality functor to the zigzag (19) and then take the homology. At the starting point we get the left-hand side of (26) and at the end we get the right-hand side. Notice that this corollary describes the rational homology of certain configuration spaces of points in suspensions.

Now notice that the sequences $\tilde{H}_{*}\left(\Sigma Y_{*}\right)^{\otimes \bullet}$ and $\tilde{H}_{*}\left(\left(\Sigma Y_{*}\right)^{\wedge} \bullet / \Delta^{\bullet} \Sigma Y_{*}\right)$ are naturally left modules over the commutative operad Com. Indeed, the first one is freely generated by its arity one component $\tilde{H}_{*}\left(\Sigma Y_{*}\right)^{\otimes 1}$, while the left Com-module structure on the second one is induced by the maps

$$
\left(\left(\Sigma Y_{*}\right)^{\wedge m} / \Delta^{m} \Sigma Y_{*}\right) \wedge\left(\left(\Sigma Y_{*}\right)^{\wedge n} / \Delta^{n} \Sigma Y_{*}\right) \longrightarrow\left(\left(\Sigma Y_{*}\right)^{\wedge m+n} / \Delta^{m+n} \Sigma Y_{*}\right)
$$

(More generally if a right $\mathrm{Com}_{+}$-module has a compatible left action by another operad $\mathcal{O}$, then its Koszul dual also naturally is a left $\mathrm{O}$-module.)

Proposition 5.3. The isomorphism (26) respects the left Com action.

Proof. It is enough to check that each map in the zigzag (19) respects the left Com action.

5.1. Complexes $\mathrm{CH}^{\Sigma Y_{*}}\left(L_{*}\right)$. Proof of Theorem 4, We define complexes $\mathrm{CH}^{\Sigma Y_{*}}\left(L_{*}\right)$ as follows

$$
\begin{aligned}
\operatorname{Rmod}_{\mathrm{Com}_{+}}\left(\tilde{H}_{*}\left(\left(\Sigma Y_{*}\right)^{\wedge} / \Delta^{\bullet} \Sigma Y_{*}\right) \circ \operatorname{Com}_{+}, \operatorname{cr} L_{*}\right) & \simeq \\
& \left(\prod_{n \geq 0} \operatorname{Hom}_{n}\left(\tilde{H}_{*}\left(\left(\Sigma Y_{*}\right)^{\wedge n} / \Delta^{n} \Sigma Y_{*}\right), \operatorname{cr} L_{*}(n)\right), d_{Y_{*}}+d_{L_{*}}\right),
\end{aligned}
$$

where $d_{L_{*}}$ is the part of the differential induced by the differential in $L_{*}$, and $d_{Y_{*}}$ is induced by the

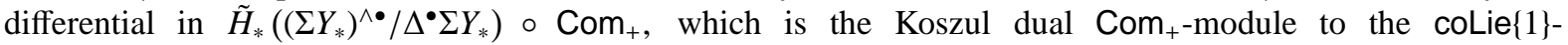
comodule $\tilde{H}_{*}\left(\left(\Sigma Y_{*}\right)^{\wedge} / \Delta^{\bullet} \Sigma Y_{*}\right)$. Explicitly, if $f \in \operatorname{Hom}_{n}\left(\tilde{H}_{*}\left(\left(\Sigma Y_{*}\right)^{\wedge n} / \Delta^{n} \Sigma Y_{*}\right), \operatorname{cr} L_{*}(n)\right)$, one has $d_{Y_{*}} f \in$ $\operatorname{Hom}_{n+1}\left(\tilde{H}_{*}\left(\left(\Sigma Y_{*}\right)^{\wedge n+1} / \Delta^{n+1} \Sigma Y_{*}\right), \operatorname{cr} L_{*}(n+1)\right)$ is defined as follows

$$
\left(d_{Y_{*}} f\right)\left(\gamma\left(x_{1} \ldots x_{n+1}\right)\right)=\sum_{1 \leq i<j \leq n} f\left(\gamma_{i j}\left(x_{1} \ldots x_{i \sim j} \ldots x_{n}\right)\right) \circ_{i \sim j}\left(x_{i} \cdot x_{j}\right),
$$

where $\gamma_{i j}$ is computed from the formula $\circ_{i \sim j}(\gamma)=\gamma_{i j} \otimes\left[x_{i}, x_{j}\right]^{\vee}$ of the coLie $\{1\}$ coaction.

Now we check that $\mathrm{CH}^{(-)}\left(L_{*}\right)$ satisfies the properties from Theorem 4 Firstly, $\mathrm{CH}^{(-)}\left(L_{*}\right):\left.\operatorname{Top}_{*}\right|_{\Sigma} \rightarrow d g V e c t$ is a well defined functor: a pointed map $\Sigma Y_{*} \rightarrow \Sigma Z_{*}$ induces a map of coLie\{1\}-comodules

$$
\tilde{H}_{*}\left(\left(\Sigma Y_{*}\right)^{\wedge} / \Delta^{\bullet} \Sigma Y_{*}\right) \rightarrow \tilde{H}_{*}\left(\left(\Sigma Z_{*}\right)^{\wedge \bullet} / \Delta^{\bullet} \Sigma Z_{*}\right) .
$$

It computes the Hochschild-Pirashvili homology functor by the coformality property, see Proposition 5.2 . Using isomorphism (26) we can define the $m$-th truncation of $\tilde{H}_{*}\left(\left(\Sigma Y_{*}\right)^{\wedge} / \Delta^{\bullet} \Sigma Y_{*}\right)$ as the cofree part cogenerated by $\tilde{H}_{*}\left(\Sigma Y_{*}\right)^{\otimes i}, i \leq m$. In the Hochschild homology this obviously corresponds to the Hodge filtration defined in Subsection 4.2. The map of graded quotients is determined by the morphism in homology $f_{*}: \tilde{H}_{*}(\Sigma Y) \rightarrow \tilde{H}_{*}(\Sigma Z)$ due to Corollary 1 and Proposition 5.3 (see also next section, where this is shown more explicitly). The splitting of the Hodge filtration over $\Sigma\left(\operatorname{Top}_{*}\right)$ has been shown in the previous section.

Now let us check that the complexes $\mathrm{CH}^{\vee_{n} S^{1}}\left(L_{*}\right)$ coincide with $C H^{\vee_{n} S^{1}}\left(L_{*}\right)$ defined in Section 3 , To see this one needs to identify $\operatorname{cr} L_{*}(\bullet)$ with $N L_{*}(\bullet)$ by means of the isomorphism (14). For simplicity let us start with the case $n=1$. One has $\left(S^{1}\right)^{\wedge k} / \Delta^{k} S^{1}=\vee_{k !} S^{k}$. Thus,

$$
\prod_{k \geq 0} \operatorname{Hom}_{k}\left(\tilde{H}_{*}\left(\left(S^{1}\right)^{\wedge k} / \Delta^{k} S^{1}\right), N L_{*}(k)\right)=\prod_{k=0}^{+\infty} N L_{*}(k)[k]=\operatorname{Tot} L_{*} \circ\left(S^{1}\right) .
$$

One can check that the differentials agree. In case of arbitrary $n$, one has $\left(\vee_{n} S^{1}\right)^{\wedge k} / \Delta^{k}\left(\vee_{n} S^{1}\right)=\vee_{k_{1}+\ldots+k_{n}=k} \vee_{k !} S^{k}$, and one similarly gets

$$
\prod_{k \geq 0} \operatorname{Hom}_{k}\left(\tilde{H}_{*}\left(\left(\vee_{n} S^{1}\right)^{\wedge k} / \Delta^{k}\left(\vee_{n} S^{1}\right)\right), N L_{*}(k)\right)=\prod_{k=0}^{+\infty} \prod_{k_{1}+\ldots+k_{n}=k} N L_{*}(k)[k]=\operatorname{Tot}(L_{*} \circ\left(\vee_{n} S^{1}\right) \underbrace{\bullet \ldots \bullet}_{n}) .
$$

For the last identity, see equation (11).

Remark 5.4. The monoid $\operatorname{End}\left(F_{n}\right)$ describes the homotopy classes of poined self-maps $\vee_{n} S^{1} \rightarrow \vee_{n} S^{1}$ and thus acts on the coLie $\{1\}$-comodule $\tilde{H}_{*}\left(\left(\vee_{n} S^{1}\right)^{\wedge} \bullet / \Delta^{\bullet}\left(\vee_{n} S^{1}\right)\right)$. One can check that the induced action on $\mathrm{CH}^{\vee_{n} S^{1}}\left(L_{*}\right)$ coincides with the one on $C H^{\vee_{n} S^{1}}\left(L_{*}\right)$ described explicitly in Section 3 . 


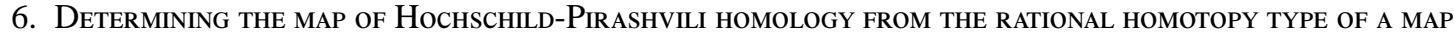

It is clear from the definition that the rational homology type of a space determines the rational higher Hochschild homology. In other words, if $X_{*} \rightarrow W_{*}$ is a rational homology equivalence then the induced map $H H^{W_{*}}\left(L_{*}\right) \rightarrow H H^{X_{*}}\left(L_{*}\right)$ is an isomorphism. Similarly, the rational homology type of any map $X_{*} \rightarrow W_{*}$ determines the map in rational Hochschild-Pirashvili homology. In particular, the rational homotopy type of a map must determine the higher Hochschild homology map. (In fact for suspensions the rational homology and rational homotopy equivalences are the same.) In this section we compute how exactly the map of suspensions induces the map of Hochschild complexes. For simplicity we will be assuming that the homology groups of the spaces that we consider are of finite type. Many of the results hold without this restriction, but require more technical work involving careful colimit arguments. Since the goal is to make it applicable for concrete examples which in practice always have this property, we concentrate on this case.

6.1. Determining the map of Koszul duals from the rational homotopy type of a map. First we need to understand how the map of Koszul duals

$$
\tilde{H}_{*}\left(\left(\Sigma Y_{*}\right)^{\wedge \bullet} / \Delta^{\bullet} \Sigma Y_{*}\right) \rightarrow \tilde{H}_{*}\left(\left(\Sigma Z_{*}\right)^{\wedge \bullet} / \Delta^{\bullet} \Sigma Z_{*}\right) .
$$

is determined by the rational homotopy type of a map $f: \Sigma Y_{*} \rightarrow \Sigma Z_{*}$. Any such map produces a commutative square of right coLie $\{1\}$-comodules:

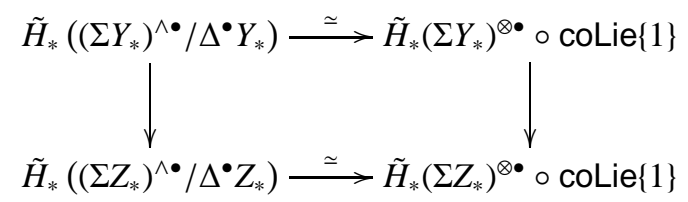

The horizontal arrows are the isomorphisms from Corollary 1 We are interested in the right vertical map. (Notice that since $f$ is arbitrary and not necessarily a suspension, this right vertical map is not determined by the induced map in homology $f_{*}: \tilde{H}_{*}\left(\Sigma Y_{*}\right) \rightarrow \tilde{H}_{*}\left(\Sigma Z_{*}\right)$.) According to Proposition 5.3, the horizontal maps respect the left Com action. It is quite obvious that the left vertical map does so as well. As a consequence, the right vertical map also respects this action. Its source is freely generated as a left Com-module by $\tilde{H}_{*}\left(\Sigma Y_{*}\right)^{\otimes 1} \circ$ coLie $\{1\}$, and its target is cofreely cogenerated as a coLie $\{1\}$ right comodule by $\tilde{H}_{*}\left(\Sigma Z_{*}\right)^{\otimes \bullet}$. As a consequence this map is determined by a map of symmetric sequences

$$
\tilde{H}_{*}\left(\Sigma Y_{*}\right)^{\otimes 1} \circ \text { coLie }\{1\} \longrightarrow \tilde{H}_{*}\left(\Sigma Z_{*}\right)^{\otimes \bullet}
$$

or equivalently by a map

$$
\tilde{H}_{*}\left(Y_{*}\right) \rightarrow \operatorname{FreeLie}\left(\tilde{H}_{*} Z_{*}\right)
$$

where FreeLie $\left(\tilde{H}_{*} Z_{*}\right)$ denotes the free completed Lie algebra generated by $\tilde{H}_{*} Z_{*}$.

The rational homotopy of a simply connected suspension is a free Lie algebra generated by its reduced homology. We claim that in the simply connected case the map obtained in (29) describes exactly the map (of generators) of rational homotopy. More generally, when the suspensions are not necessarily simply connected, one can still assign a morphism (29) to the rational homotopy type of a map $f: \Sigma Y_{*} \rightarrow \Sigma Z_{*}$. By Lemma4.2 any suspension is rationally formal. Thus the induced map of their Sullivan's models

$$
A_{\Sigma Z_{*}} \rightarrow A_{\Sigma Y_{*}}
$$

is quasi-isomorphic to a map of dg algebras

$$
\mathcal{A}\left(\mathcal{L}^{c}\left(\tilde{H}^{*} \Sigma Z_{*}\right)\right) \rightarrow H^{*} \Sigma Y_{*},
$$

where the left-hand side is the cofibrant replacement of $H^{*} \Sigma Z_{*}$ obtained as the Chevalley-Eilenberg complex $\mathcal{A}(-)$ of the Harrison complex $\mathcal{L}^{c}(-)$ of the (non-unital) algebra $\tilde{H}^{*} \Sigma Z_{*}$. Notice that $\mathcal{L}^{c}\left(\tilde{H}^{*} \Sigma Z_{*}\right)$ is the cofree Lie coalgebra cogenerated by $\tilde{H}^{*} Z_{*}$ (with zero differential). Its dual vector space is exactly FreeLie $\left(\tilde{H}_{*} Z_{*}\right)$. The map of algebras (30) is determined by its restriction on the space of generators

$$
\mathcal{L}^{c}\left(\tilde{H}^{*} \Sigma Z_{*}\right) \rightarrow \tilde{H}^{*} \Sigma Y_{*} .
$$

Proposition 6.1. For any map $f: \Sigma Y_{*} \rightarrow \Sigma Z_{*}$ of pointed suspensions of finite type, the map (31) encoding the rational homotopy type of $f$ is dual to the map (29) encoding the homotopy type of the induced map of right $\mathrm{Com}_{+}$ modules

$$
\tilde{C}_{*}\left(\left(\Sigma Y_{*}\right)^{\wedge \bullet}\right) \rightarrow \tilde{C}_{*}\left(\left(\Sigma Z_{*}\right)^{\wedge \bullet}\right) .
$$




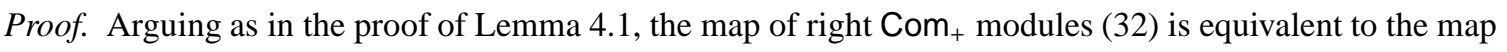

$$
\left(\tilde{H}_{*} \Sigma Y_{*}\right)^{\otimes \bullet} \rightarrow\left(\tilde{\mathcal{A}}\left(\mathcal{L}^{c}\left(\tilde{H}^{*} \Sigma Z_{*}\right)\right)^{\otimes \bullet}\right)^{\vee},
$$

where $\tilde{\mathcal{A}}(-)$ denotes the augmented part of $\mathcal{A}(-)$; “V” denotes taking the dual of a graded vector space. The map (33) in each arity is the dual of a tensor power of (30). The right-hand side of (33) can also be expressed as $\left(\tilde{\hat{\mathbf{C}}}\left(\operatorname{FreeLie}\left(\tilde{H}_{*} Z_{*}\right)\right)\right)^{\hat{\otimes} \bullet}$, where $\tilde{\hat{\mathbf{C}}}(-)$ denotes the completed augmented Chevalley-Eilenberg complex (of a completed Lie algebra FreeLie $\left.\left(\tilde{H}_{*} Z_{*}\right)\right)$; “ $\hat{\otimes} ”$ denotes the completed tensor product.

One has a zigzag of right $\mathrm{Com}_{+}$-modules

$$
\left(\tilde{H}_{*} \Sigma Y_{*}\right)^{\otimes \bullet} \rightarrow\left(\tilde{\hat{\mathbf{C}}}\left(\operatorname{FreeLie}\left(\tilde{H}_{*} Z_{*}\right)\right)\right)^{\hat{\otimes} \bullet} \stackrel{\simeq}{\longleftarrow}\left(\tilde{H}_{*} \Sigma Z_{*}\right)^{\otimes \bullet},
$$

where the right arrow is an equivalence. We get a zigzag of their Koszul duals:

$$
\left(\tilde{H}_{*} \Sigma Y_{*}\right)^{\otimes \bullet} \circ \operatorname{coLie}\{1\} \rightarrow\left(\tilde{\hat{\mathbf{C}}}\left(\operatorname{FreeLie}\left(\tilde{H}_{*} Z_{*}\right)\right)\right)^{\hat{\otimes} \bullet} \circ \operatorname{coLie}\{1\} \stackrel{\simeq}{\longleftarrow}\left(\tilde{H}_{*} \Sigma Z_{*}\right)^{\otimes \bullet} \circ \operatorname{coLie}\{1\},
$$

We claim that the right arrow has a natural left inverse. In order to construct this left inverse

$$
\left(\tilde{\hat{\mathbf{C}}}\left(\operatorname{FreeLie}\left(\tilde{H}_{*} Z_{*}\right)\right)\right)^{\hat{\otimes} \bullet} \circ \operatorname{coLie}\{1\} \stackrel{\simeq}{\longrightarrow}\left(\tilde{H}_{*} \Sigma Z_{*}\right)^{\otimes \bullet} \circ \operatorname{coLie}\{1\}
$$

it is enough to define a map of their (co)generators

$$
\left(\tilde{\hat{\mathbf{C}}}\left(\operatorname{FreeLie}\left(\tilde{H}_{*} Z_{*}\right)\right)\right)^{\hat{\otimes} 1} \circ \operatorname{coLie}\{1\} \longrightarrow\left(\tilde{H}_{*} \Sigma Z_{*}\right)^{\otimes \bullet} .
$$

In arity $n$ the latter map of symmetric sequences is defined as the following composition

$$
\begin{aligned}
& \tilde{\hat{\mathbf{C}}}\left(\operatorname{FreeLie}\left(\tilde{H}_{*} Z_{*}\right)\right) \otimes \operatorname{coLie}\{1\}(n) \rightarrow \operatorname{FreeLie}\left(\tilde{H}_{*} Z_{*}\right)[-1] \otimes \operatorname{coLie}\{1\}(n) \rightarrow \\
& \qquad \operatorname{Lie}(n) \otimes_{n}\left(\tilde{H}_{*} \Sigma Z_{*}\right)^{\otimes n} \otimes \operatorname{coLie}(n) \rightarrow\left(\tilde{H}_{*} \Sigma Z_{*}\right)^{\otimes n} .
\end{aligned}
$$

The first map is induced by the projection on cogenerators $\tilde{\hat{\mathbf{C}}}\left(\operatorname{FreeLie}\left(\tilde{H}_{*} Z_{*}\right)\right) \rightarrow \operatorname{FreeLie}\left(\tilde{H}_{*} Z_{*}\right)[-1]$. The second map is obtained by projecting FreeLie $\left(\tilde{H}_{*} Z_{*}\right)$ onto its subspace spanned by brackets of length $n$. The last map takes into account the duality between the spaces Lie $(n)$ and coLie $(n)$ :

$$
L \otimes h_{1} \otimes \ldots \otimes h_{n} \otimes L^{\prime} \mapsto \sum_{\sigma \in \epsilon_{n}}\left(\sigma L, L^{\prime}\right) h_{\sigma_{1}} \otimes \ldots \otimes h_{\sigma_{n}} .
$$

To finish the proof we notice that the composite of the first arrow in (34) and the constructed inverse is the map

$$
\left(\tilde{H}_{*} \Sigma Y_{*}\right)^{\otimes \bullet} \circ \operatorname{coLie}\{1\} \rightarrow\left(\tilde{H}_{*} \Sigma Z_{*}\right)^{\otimes \bullet} \circ \operatorname{coLie}\{1\}
$$

(co)generated by the map dual do $\underline{31}$.

6.2. Determining map of Hochschild-Pirashvili homology. In this subsection we describe how the map

$$
\tilde{H}_{*} Y_{*} \rightarrow \operatorname{FreeLie}\left(\tilde{H}_{*} Z\right)
$$

encoding the rational homotopy type of $f: \Sigma Y_{*} \rightarrow \Sigma Z_{*}$, determines the map of higher Hochschild complexes $\mathrm{CH}^{(-)}(-)$(in fact we will work with $g r \mathrm{CH}^{(-)}(-)$instead). For simplicity we will be assuming that $Y_{*}$ and $Z_{*}$ are of finite type and we will only look at the case $L_{*}=M \otimes \mathbf{C}(\mathfrak{g})^{\otimes \bullet}$, where $\mathfrak{g}$ is strictly positively graded. Thus we need to describe the induced map

$$
M \hat{\otimes} S\left(\tilde{H}^{*} Z_{*} \hat{\otimes} \mathfrak{g}\right) \rightarrow M \hat{\otimes} S\left(\tilde{H}^{*} Y_{*} \hat{\otimes} \mathfrak{g}\right) .
$$

Firstly, this map is the tensor product of the identity on the first factor $M$ and a coalgebra homomorphism on the second one. Ergo, it's enough to describe its composition with the projection to the space of cogenerators

$$
S\left(\tilde{H}^{*} Z_{*} \hat{\otimes} \mathfrak{g}\right) \rightarrow \tilde{H}^{*} Y_{*} \hat{\otimes} \mathfrak{g}
$$

The map (35) is a product of maps

$$
\tilde{H}_{*} Y \rightarrow \operatorname{Lie}(n) \otimes_{n}\left(\tilde{H}_{*} Z_{*}\right)^{\otimes n} .
$$

its $n$-th component $(38)$ can be viewed as an element $\rho_{n} \in \tilde{H}^{*} Y_{*} \hat{\otimes} \operatorname{Lie}(n) \otimes_{n}\left(\tilde{H}_{*} Z\right)^{\otimes n}$. This element $\rho_{n}$ contributes only to

$$
S^{n}\left(\tilde{H}^{*} Z_{*} \otimes \mathfrak{g}\right) \rightarrow \tilde{H}^{*} Y_{*} \otimes \mathfrak{g}
$$


in (37). The element $\rho_{n}$ is a sum of elements of the form

$$
h^{0} \otimes L \otimes h_{1} \otimes \ldots \otimes h_{n} \in \tilde{H}^{*} Y_{*} \otimes \operatorname{Lie}(n) \otimes{ }_{n}\left(\tilde{H}_{*} Z\right)^{\otimes n} .
$$

Each such summand contributes to $(39)$ as a map sending

$$
\left(h^{1} \otimes g_{1}\right) \cdot \ldots \cdot\left(h^{n} \otimes g_{n}\right) \in S^{n}\left(\tilde{H}^{*} Z_{*} \otimes \mathfrak{g}\right)
$$

to

$$
\sum_{\sigma \in \epsilon_{n}} \pm\left(\prod_{i=1}^{m}\left(h_{i}, h^{\sigma_{i}}\right)\right) h^{0} \otimes L\left(g_{\sigma_{1}}, \ldots, g_{\sigma_{n}}\right) \in \tilde{H}^{*} Y_{*} \hat{\otimes} \mathfrak{g}
$$

where the sign is as usual the Koszul one induced by permutation of elements.

In the examples below we will be omiting the hat sign over the tensor product as the induced map (36) can always be restricted on the non-completed part $M \otimes S\left(\tilde{H}^{*}(-) \otimes \mathfrak{g}\right.$ ) (where the symmetric power is also taken in the non-completed sense.)

Example 6.2. Consider the map $S^{1} \rightarrow S^{1} \vee S^{1}$ which sends the generator $x$ of $\pi_{1} S^{1}$ to the product $y_{1} y_{2}$ of generators of $\pi_{1}\left(S^{1} \vee S^{1}\right)$. The map $(35)$ becomes

$$
x \mathbb{Q} \rightarrow \operatorname{FreeLie}\left(y_{1}, y_{2}\right),
$$

that encodes the map of the primitive part of the Malcev completions [10] (all generators $x, y_{1}, y_{2}$ are of degree zero). The image of $x$ is described by the Baker-Campbell-Hausdorff formula

$$
x \mapsto \ln \left(e^{y_{1}} \cdot e^{y_{2}}\right) .
$$

The map (36) becomes

$$
M \otimes S(\mathfrak{g}) \otimes S(\mathfrak{g}) \rightarrow M \otimes S(\mathfrak{g})
$$

which sends

$$
m \otimes A \otimes B \mapsto m \otimes A \star B,
$$

where $\star$ is the associative (star) product on $S(\mathfrak{g})$ transported from $\mathcal{U}_{\mathfrak{g}}$ via the Poincaré-Birkhoff-Witt isomorphism.

Example 6.3. Consider the map $S^{2} \rightarrow S^{1} \vee S^{2}$ corresponding to the element $x \cdot y \in \pi_{2}\left(S^{1} \vee S^{2}\right)$, where $x$ is the generator of $\pi_{1} S^{1}$ and $y$ is the generator of $\pi_{2} S^{2}$. The map (35) in our case is

$$
y \mathbb{Q} \rightarrow \operatorname{FreeLie}(x, y),
$$

where $|x|=0,|y|=1$,

$$
y \mapsto e^{a d_{x}}(y)
$$

The induced map (36) is

$$
M \otimes S(\mathfrak{g}) \otimes S(\mathfrak{g}[1]) \rightarrow M \otimes S(\mathfrak{g}[1]),
$$

sending

$$
m \otimes g_{1} \cdot \ldots \cdot g_{k} \otimes s^{-1} g_{1}^{\prime} \cdot \ldots \cdot s^{-1} g_{k^{\prime}}^{\prime} \mapsto m \otimes \frac{1}{k !} \sum_{\sigma \in \epsilon_{k}} a d_{g_{\sigma_{1}}} \ldots a d_{g_{\sigma_{k}}}\left(s^{-1} g_{1}^{\prime} \cdot \ldots \cdot s^{-1} g_{k^{\prime}}^{\prime}\right) .
$$

Example 6.4. Consider the Hopf map $S^{3} \rightarrow S^{2}$. On the level of rational homotopy we get a map

$$
y \mathbb{Q} \rightarrow \operatorname{FreeLie}(x),
$$

where $|x|=1,|y|=2$, and

$$
y \mapsto \frac{1}{2}[x, x] .
$$

The induced map of higher Hochschild complexes

$$
M \otimes S(\mathfrak{g}[1]) \rightarrow M \otimes S(\mathfrak{g}[2])
$$

sends

$$
\begin{gathered}
m \otimes s^{-1} g_{1} \cdot \ldots \cdot s^{-1} g_{2 k-1} \mapsto 0, \\
m \otimes s^{-1} g_{1} \cdot \ldots \cdot s^{-1} g_{2 k} \mapsto m \otimes \frac{1}{2^{k} k !} \sum_{\sigma \in 2 k} \pm s^{-2}\left[g_{\sigma_{1}}, g_{\sigma_{2}}\right] \cdot \ldots \cdot s^{-2}\left[g_{\sigma_{2 k-1}}, g_{\sigma_{2 k}}\right] .
\end{gathered}
$$




\section{HochSCHILD-PIRASHVILI HOMOLOGY FOR NON-SUSPENSIONS}

Some of the techniques given in the present paper can also be applied to study the higher Hochschild homology for non-suspensions and maps between them. This section is a short note on how this works in the special case when $L_{*}=M \otimes \mathbf{C}(\mathfrak{g})^{\otimes \bullet}$, where $\mathrm{g}$ as usual is a strictly positively graded dg Lie algebra, and the spaces are connected and of finite type.

Theorem 5. Assuming a pointed space $X_{*}$ is connected and of finite type, let A be an augmented non-positively graded augmented commutative dg algebra of finite type quasi-isomorphic to the Sullivan algebra $A_{X_{*}}$, and $\tilde{A}$ be its augmentation ideal ${ }^{13}$ Then the Hochschild-Pirashvili homology $\operatorname{HH}^{X_{*}}(\mathbf{C}(\mathrm{g}), M)$ is computed by the complex $M \hat{\otimes} \hat{\mathbf{C}}(\tilde{A} \hat{\otimes} \mathfrak{g})$, where $\hat{\mathbf{C}}(\tilde{A} \hat{\otimes} \mathfrak{g})$ is the completed (with respect to the total homological degree of elements from $\mathfrak{g}$ ) Chevalley-Eilenberg complex of the completed Lie algebra Ã $\hat{\otimes} g$. The differential has the form

$$
d=d_{M}+d_{\mathfrak{g}}+d_{A}+d_{C E}+\delta,
$$

where $d_{M}, d_{\mathfrak{g}}, \delta$ are as those from (7), $d_{A}$ is induced by the differential in $A, d_{C E}$ is the Chevalley-Eilenberg differential.

Proof. This complex is constructed in the same way as the higher Hochschild complexes for suspensions, see Subsection 4.1 The extra term $d_{C E}$ in the differential appears due to the fact that the $\mathrm{Com}_{+}$action on $\left(\tilde{A}^{\vee}\right)^{\otimes \bullet}$ is now non-trivial.

The result of this theorem is partially known to experts. It appeared explicitly for spheres and surfaces respectively in [14, Theorem 3] and [15, Theorem 4.3.3], see also [3] for a similar implicit statement in case $X$ is a manifold. Notice also that in case $M=\mathbf{C}(\mathfrak{g})$ (i.e., when considering unpointed version of higher Hochschild homology) the obtained higher Hochschild complex is the completed Chevalley-Eilenberg complex $\hat{\mathbf{C}}(A \hat{\otimes} \mathfrak{g})$. As application of this example, in case the dimension of $X$ is less than the connectivity of $Y$, the space $Y^{X}$ of continuous maps $Y \rightarrow X$ has homology with any coefficients described as $H_{*}\left(Y^{X}\right) \simeq H H^{X}\left(C_{*}\left(Y^{\bullet}\right)\right.$ ), see [26, 28]. On the other hand, the rational homotopy type of $Y^{X}$ is described by the dg Lie algebra $A \hat{\otimes} L$, where $A$ is a suitable Sullivan model for $X$ and $L$ is a suitabe Quillen model for $Y$, see [4, 6, 7]. From this we also recover that $\hat{\mathbf{C}}(A \hat{\otimes} L)$, i.e., our complex, computes the rational homology of $Y^{X}$.

Remark 7.1. One can easily see that the $m$ th term of the Hodge filtration in $M \hat{\otimes} \hat{\mathbf{C}}(\tilde{A} \hat{\otimes} \mathfrak{g})=\prod_{i=0}^{+\infty} M \hat{\otimes} S^{i}(\tilde{A}[-1] \hat{\otimes} \mathfrak{g})$ is $F_{m} M \hat{\otimes} \hat{\mathbf{C}}(\tilde{A} \hat{\otimes} \mathfrak{g})=\prod_{i=0}^{m} M \hat{\otimes} S^{i}(\tilde{A}[-1] \hat{\otimes} \mathfrak{g})$.

Theorem 5 applied to a suspension $\Sigma Y_{*}$ of a finite type is exactly the statement of Remark 4.6 Indeed, since $\Sigma Y_{*}$ is formal one can take $\tilde{A}=\tilde{H}^{*} \Sigma Y_{*}$ the cohomology algebra, whose product is trivial, and thus the ChevalleyEilenberg part of the differential is trivial $d_{C E}=0$. The rational homotopy type of a map of suspensions of finite type $f: \Sigma Y_{*} \rightarrow: \Sigma Z_{*}$ is encoded by a map [31], which is essentially the same as a $C_{\infty} m_{\infty}$ map of commutative algebras $f_{\infty}^{*}: \tilde{H}^{*} \Sigma Z_{*} \rightarrow \tilde{H}^{*} \Sigma Y_{*}$. In Subsection 6.2 we show how this map determines a map of higher Hochschild complexes

$$
M \hat{\otimes} \hat{\mathbf{C}}\left(\tilde{H}^{*} \Sigma Z_{*} \hat{\otimes} \mathfrak{g}\right) \rightarrow M \hat{\otimes} \hat{\mathbf{C}}\left(\tilde{H}^{*} \Sigma Y_{*} \hat{\otimes} \mathfrak{g}\right),
$$

which is the identity on the first factor $M$ and a completed coalgebras map on the second factor. The latter map can be regarded as a completed $L_{\infty}$ morphism

$$
\tilde{H}^{*} \Sigma Z_{*} \hat{\otimes} \mathfrak{g} \rightarrow \tilde{H}^{*} \Sigma Y_{*} \hat{\otimes} \mathfrak{g} .
$$

of (completed) abelian Lie algebras.

More generally, a tensor product with a dg Lie algebra is in fact a functor from $\mathrm{Com}_{\infty}$ algebras to $L_{\infty}$ algebras. We will need a completed version of this construction. Let $\tilde{A}$ be a negatively graded $\mathrm{Com}_{\infty}$ algebra of finite type encoding the rational homotopy type of a connected pointed space $X_{*}$, and let $\mathrm{g}$ be a positively graded dg Lie algebra. The completed $L_{\infty}$ algebra structure on $\tilde{A} \hat{\otimes} \mathfrak{g}$ is explicitly described by the structure maps $\mu_{n}$ defined as composition

$$
\mu_{n}: S^{n}(\tilde{A}[-1] \hat{\otimes} \mathfrak{g}) \rightarrow \operatorname{FreeLie}^{c}(\tilde{A}[-1]) \hat{\otimes} \operatorname{FreeLie}(\mathfrak{g}) \rightarrow \tilde{A} \hat{\otimes} \mathfrak{g},
$$

where FreeLie ${ }^{c}(\tilde{A}[-1])$ is the free Lie coalgebra cogenerated by $A[-1]$ (in other words, it is the Harrison complex $\left.\mathcal{L}^{c}(\tilde{A})\right)$. The first map is induced by the diagonal $\operatorname{Com}(n) \rightarrow \operatorname{coLie}(n) \otimes \operatorname{Lie}(n)$. The second map is the $\operatorname{Com}_{\infty}$ structure on the first factor and the Lie structure on the second. If $\tilde{B} \rightarrow \tilde{A}$ is a Com $_{\infty}$ morphism encoding the rational homotopy type of a pointed map $X_{*} \rightarrow Y_{*}$, then the induced completed $L_{\infty}$ map $\tilde{B} \hat{\otimes} \mathfrak{g} \rightarrow \tilde{A} \otimes \hat{g}$ is described by essentially the same formulas as (41). Its $n$-th component is the composition

$$
F_{n}: S^{n}(\tilde{B}[-1] \hat{\otimes} \mathfrak{g}) \rightarrow \operatorname{FreeLie}^{c}(\tilde{B}[-1]) \hat{\otimes} \operatorname{FreeLie}(\mathfrak{g}) \rightarrow \tilde{A}[-1] \hat{\otimes} \mathfrak{g},
$$

\footnotetext{
${ }^{13}$ In our conventions all the complexes have differential of degree -1 , for which reason the algebras we consider are non-positively graded.
} 
where the first map is the same as the fist one in (41). The second map is the tensor product of the Com $_{\infty}$ map $\tilde{B} \rightarrow \tilde{A}$ and the Lie algebra structure map on $\mathfrak{g}$. In Subsection 6.2 the corresponding $L_{\infty}$ map is explained in full detail for the case of suspensions $\tilde{A}=\tilde{H}^{*} \Sigma Y_{*}, \tilde{B}=\tilde{H}^{*} \Sigma Z_{*}$.

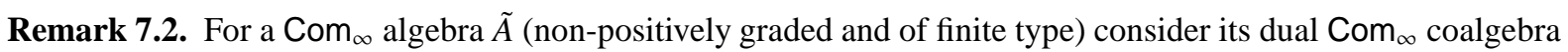
$\tilde{A}^{\vee}$. Then the $L_{\infty}$ algebra $\tilde{A} \hat{\otimes} \mathfrak{g}$ considered above is the $L_{\infty}$ algebra of derivations of the zero map of Lie algebras $\mathcal{L}\left(\tilde{A}^{\vee}\right) \rightarrow \mathfrak{g}$.

Theorem 6. Let Ã be a non-positively graded $\mathrm{Com}_{\infty}$ algebra of finite type encoding the rational homotopy type of a pointed space $X_{*}$, then the Hochschild-Pirashvili homology $H^{X_{*}}(\mathbf{C}(\mathfrak{g}), M)$ is computed by the complex $M \hat{\otimes} \hat{\mathbf{C}}(\tilde{A} \otimes \mathfrak{g})$, where $\hat{\mathbf{C}}(\tilde{A} \otimes \mathfrak{g})$ is the completed Chevalley-Eilenberg complex of the completed $L_{\infty}$ algebra $\tilde{A} \hat{\otimes} \mathrm{g}$. The differential has the form (40). If $\tilde{B} \rightarrow \tilde{A}$ is a $\mathrm{Com}_{\infty}$ morphism (of non-positively graded $\mathrm{Com}_{\infty}$ algebras of finite type) encoding the rational homotopy type of a pointed map $X_{*} \rightarrow Y_{*}$, then the induced map in the Hochschild-Pirashvili homology

$$
H H^{Y_{*}}(\mathbf{C}(\mathfrak{g}), M) \rightarrow H H^{X_{*}}(\mathbf{C}(\mathfrak{g}), M)
$$

is computed by the chain map

$$
M \hat{\otimes} \hat{\mathbf{C}}(\tilde{B} \otimes \mathfrak{g}) \rightarrow M \hat{\otimes} \hat{\mathbf{C}}(\tilde{A} \otimes \mathfrak{g}),
$$

which is identity on the first factor $M$ and a completed coalgebra map corresponding to the induced completed $L_{\infty}$ algebras map $\tilde{B} \hat{\otimes} \mathfrak{g} \rightarrow \tilde{A} \hat{\otimes} \mathfrak{g}$.

Proof. First we check that the statement of the theorem holds when $\tilde{B} \rightarrow \tilde{A}$ is a dg commutative algebras map,

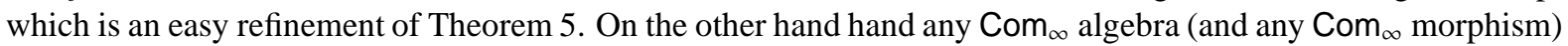
is quasi-isomorphic to a dg commutative algebra (map of dg commutative algebras). This together with the fact that a $\operatorname{Com}_{\infty}$ quasi-isomorphism $\tilde{A}_{1} \rightarrow \tilde{A}_{2}$ induces an $L_{\infty}$ quasi-isomorphism $\tilde{A}_{1} \hat{\otimes} \mathfrak{g} \rightarrow \tilde{A}_{2} \hat{\otimes} \mathfrak{g}$ proves the staement of the theorem.

The above theorem has the following corollary.

Proposition 7.3. For a pointed connected space $X_{*}$ of finite type, the Hodge filtration in the higher Hochschild complexes splits for any coefficient $\Gamma$ module $L_{*}$ if and only if $X_{*}$ is rationally homology equivalent to a suspension.

Proof. In one direction the statement easily follows from the fact that a rational homology equivalence of spaces induces a quasi-isomorphism of higher Hochschild complexes. Now let $X_{*}$ be not equivalent to a suspension. It is well known that any $\mathrm{Com}_{\infty}$ algebra is $\mathrm{Com}_{\infty}$ quasi-isomorphic to a one with zero differential [22, Theorem 10.4.5]. Let $\tilde{A}$ be such one encoding the rational homotopy type of $X_{*}$. Since we assume $X_{*}$ is not rationally a suspension, $\tilde{A}$ must have non-trivial (higher) product(s). Let $k$ be the arity of the first non-trivial product. We choose $L_{*}=M \otimes \mathbf{C}(\mathfrak{g})^{\otimes \bullet}$, where $M=\mathbb{Q}$ is the comodule with the trivial coaction, and $\mathfrak{g}$ is a free Lie algebra with $k$ generators. By construction $\tilde{A} \hat{\otimes} g$ is an $L_{\infty}$ algebra with zero differential and whose first non-trivial (higher) bracket has arity $k$. Applying Remark 7.1 we get that the $(k-1)$ th differential in the spectral sequence associated with the Hodge filtration in $M \hat{\otimes} \hat{\mathbf{C}}(\tilde{A} \otimes \mathfrak{g})$ is non-zero. Therefore the filtration does not split.

7.1. Hochschild-Pirashvili homology as "homotopy base change". Let us conclude by remarking on a curious algebraic interpretation of the Hochschild-Pirashvili homology in the form described in Theorem 5 . First, recall that to any $\mathrm{dg}$ commutative algebra $A$ we may associate a functor

$$
\Phi_{A}: \text { (Lie algebras) } \rightarrow \text { (Lie algebras) }
$$

by sending a dg Lie algebra $\mathfrak{g}$ to the tensor product $\Phi_{A}(\mathfrak{g}):=\mathfrak{g} \otimes A$, with the Lie algebra structure $A$-linearly extended in the obvious manner. We may call this functor $\Phi_{A}$ "base change", even though this is a misnomer as we do not change the underlying ground ring. Similarly, if $\mathrm{g}$ is a dg Lie algebra and $K$ is an $A$-module, we may define a functor

$$
\Psi_{A, K}:(\mathfrak{g} \text { - modules }) \rightarrow\left(\Phi_{A}(\mathfrak{g}) \text { - modules }\right)
$$

by sending a $\mathfrak{g}$-module $\mathfrak{f}$ to the $\Phi_{A}(\mathfrak{g})$-module $\Psi_{A, K}(\mathfrak{f}):=\mathfrak{f} \otimes K$, with the module structure defined in the obvious manner. We also call the functor $\Psi_{A, K}$ "base change", with the same caveat as above that this is a misnomer. There is also a topological variant: If the Lie algebra $g$ carries in addition a complete topology compatible with the Lie algebra structure, then $\hat{\Phi}_{A}(\mathfrak{g}):=\mathfrak{g} \hat{\otimes} A$ is likewise equipped with a natural complete filtration. Similarly, if $\mathfrak{f}$ is equipped with a complete filtration and the action of $A$ is continuous, then $\hat{\Psi}_{A, K}(\mathfrak{f}):=\mathfrak{f} \hat{\otimes} K$ is a complete (continuous) $\Phi_{A}(\mathrm{~g})$-module.

Now it is well known [22, chapter 11.3] that there is an adjunction of categories

$$
\mathcal{L}: \text { (conilpotent coaugmented dg cocommutative coalgebras) } \leftrightarrows(\text { dg Lie algebras) : } \mathbf{C}
$$


given by the bar and cobar functors (i.e., the Harrison and Chevalley complex functors), such that for any conilpotent dg coalgebra $C$ the unit of the adjunction $C \rightarrow \mathbf{C}(\mathcal{L}(C))$ is a quasi-isomorphism, and such that for any $\mathrm{dg}$ Lie algebra $\mathfrak{g}$ the counit of the adjunction $\mathcal{L}(\mathbf{C}(\mathfrak{g})) \rightarrow \mathfrak{g}$ is a quasi-isomorphism. Concretely, the functor $\mathcal{L}$ takes the Harrison complex (a free Lie algebra) of the cokernel of the coaugmentation, while the functor $\mathbf{C}$ takes the Chevalley complex. Similar functors exist on the the level of comodules. If $C$ is a conilpotent dg cocommutative coalgebra then we have bar and cobar functors

$$
\left.\mathcal{L}_{\text {mod }}: \text { (conilpotent } C \text {-comodules }\right) \rightarrow(\mathcal{L}(C) \text { - modules })
$$

(conilpotent $\mathbf{C}(\mathcal{L}(C))$-comodules $) \leftarrow\left(\mathcal{L}(C)\right.$ - modules) : $\mathbf{C}_{\text {mod }}$

Concretely, $\mathcal{L}_{\text {mod }}(M)=\operatorname{Harr}(C ; M)$ is the Harrison complex with values in the module $M$, i.e., a free $\mathcal{L}(C)$-module generated by $M$ if we disregard the differential. Similarly, $\mathbf{C}_{\bmod }(N)=\mathbf{C}(\mathcal{L}(C) ; N)$ is the Chevalley complex with values in $N$, i.e., a cofree $\mathbf{C}(\mathcal{L}(C)$ )-comodule cogenerated by $N$ with a natural differential.

There exist versions of the above constructions for complete topological algebras and modules, by replacing tensor products appearing there by a completed version. We denote those completed versions by $\hat{\mathcal{L}}, \hat{\mathbf{C}}$ etc.

The above adjunctions allow us to transport any endofunctor of the category of $\mathrm{dg}$ Lie algebras to an endofunctor of the category of conilpotent dg cocommutative coalgebras (and vice versa). The point of this section is to remark that the Hochschild-Pirashvili homology functor is nothing but the (homology of the) well known base change functors above, transported to the category of conilpotent coalgebras via the bar and cobar adjunctions. This gives an algebraically "very simple" interpretation of the Hochschild-Pirashvili homology. Concretely, let us assume that we are given the following data:

- A conilpotent complete cocommutative dg coalgebra $C$, for example $C=\mathbf{C}(\mathfrak{g})$, for a dg Lie algebra $\mathfrak{g}$ as in Theorem 5, which we endow with the complete the decreasing filtration by degree.

- A a conilpotent complete $C$-comodule $M$.

- An augmented dg commutative algebra $A$. For example, we may take such an $A$ from Theorem 5 , We will still denote by $\tilde{A}$ its augmentation ideal.

- We let $K=\mathbb{Q}$ be the one-dimensional $A$-module, with the action defined by the augmentation.

Then we define a complete cocommutative coalgebra

$$
C_{A}:=\hat{\mathbf{C}}\left(\hat{\Phi}_{A}(\mathcal{L}(C))\right)=\hat{\mathbf{C}}(\mathcal{L}(C) \hat{\otimes} A)
$$

and the complete $C_{A}$-comodule

$$
M_{A}:=\hat{\mathbf{C}}_{\text {mod }}\left(\hat{\Psi}_{A, K}\left(\mathcal{L}_{\text {mod }}(M)\right)\right)=\hat{\mathbf{C}}_{\text {mod }}\left(\mathcal{L}_{\text {mod }}(M) \hat{\otimes} K\right) .
$$

Clearly, these constructions are functorial in $A, C$ and $M$. We will abusively call these constructions "homotopy base change". The main statement of this section is then that the complex of Theorem 5 computing the HochschildPirashvili homology may be interpreted as "homotopy base change".

Proposition 7.4. For $C=\mathbf{C}(\mathfrak{g})$ the Chevalley complex of a dg Lie algebra, and $A, M$ as above, the complexes $M_{A}$ and the complex $(M \hat{\otimes} \hat{\mathbf{C}}(\tilde{A} \hat{\otimes} \mathfrak{g}), d)$ of Theorem 5 are quasi-isomorphic.

Proof. Explicitly, the complex $M_{A}$ has the form

$$
\hat{\mathbf{C}}(\mathcal{L}(C) \hat{\otimes} A ; \operatorname{Harr}(C ; M) \otimes K)
$$

where $\hat{\mathbf{C}}(-;-)$ denotes the (completed) Chevalley complex with values in the second argument, and $\operatorname{Harr}(-;-)$ denotes the Harrison complex. Using the augmentation we may now split $A=\mathbb{Q} \oplus \tilde{A}$, where $\tilde{A}$ is the kernel of the augmentation. Using this splitting we find the identification of graded vector spaces (recall that $K=\mathbb{Q}$ )

$$
\hat{\mathbf{C}}(\mathcal{L}(C) \hat{\otimes} A ; \operatorname{Harr}(C ; M) \otimes K) \cong \hat{\mathbf{C}}(\mathcal{L}(C) \hat{\otimes} \tilde{A}) \hat{\otimes} \mathbf{C}(\mathcal{L}(C) ; \operatorname{Harr}(C ; M)) \text {. }
$$

Note however, that this identification is not an identification of complexes (yet). The differential on the right-hand side is composed of two terms: the differential $d_{1}$ of the left-hand tensor factor and the differential $d_{2}$ of the righthand tensor factor. The differential on the left-hand side of (43) on the other hand has an additional term $d_{\text {mixed }}$ from the Chevalley differential, which is obtained by taking the coaction of $\mathbf{C}(\mathcal{L}(C)$; $\operatorname{Harr}(C ; M))$ followed by a Lie bracket. Note that this term resembles the term $\delta$ in Theorem 5 Note that we have a quasi-isomorphism of $\mathbf{C}(\mathcal{L}(C))$-comodules

$$
M \rightarrow \mathbf{C}(\mathcal{L}(C) ; \operatorname{Harr}(C ; M)) .
$$

Hence we obtain a quasi-isomorphism of complexes

$$
\left(\hat{\mathbf{C}}(\mathcal{L}(C) \hat{\otimes} \tilde{A}) \otimes M, d_{1}+d_{M}+d_{\text {mixed }}\right) \stackrel{\sim}{\rightarrow}\left(\hat{\mathbf{C}}(\mathcal{L}(C) \hat{\otimes} \tilde{A}) \hat{\otimes} \mathbf{C}(\mathcal{L}(C) ; \operatorname{Harr}(C ; M)), d_{1}+d_{2}+d_{\text {mixed }}\right) \cong M_{A},
$$

where the part of the differential $d_{\text {mixed }}$ on the left-hand complex is defined as before by taking the coaction on $M$ followed by a Lie bracket with a factor of $\hat{\mathbf{C}}(\mathcal{L}(C) \hat{\otimes} \tilde{A})$. 
Furthermore, since $C=\mathbf{C}(\mathfrak{g})$ we have a quasi-isomorphism of dg Lie algebras

$$
\mathcal{L}(C) \rightarrow \mathfrak{g} .
$$

Hence we obtain a quasi-isomorphism

$$
\left(\hat{\mathbf{C}}(\mathcal{L}(C) \hat{\otimes} \tilde{A}) \hat{\otimes} M, d_{1}+d_{M}+d_{\text {mixed }}\right) \stackrel{\sim}{\rightarrow}(\hat{\mathbf{C}}(\mathrm{g} \hat{\otimes} \tilde{A}) \hat{\otimes} M, d)
$$

with the complex considered in Theorem 5 By (44) and (45) the Proposition is shown.

\section{REFERENCES}

[1] G. Arone, V. Turchin. On the rational homology of high dimensional analogues of spaces of long knots. Geom. Topol. 18 (2014) 12611322.

[2] G. Arone, V. Turchin. Graph-complexes computing the rational homotopy of high dimensional analogues of spaces of long knots. Annales de l'Institut Fourier 65 (2015), no. 1, 1-62.

[3] D. Ayala, J. Francis. Factorization homology of topological manifolds. J. Topol. 8 (2015), no 4, 1045-1084.

[4] J. Block, A. Lazarev. André-Quillen cohomology and rational homotopy of function spaces. Adv. in Math. 193 (2005), 18-39.

[5] M. R. Bridson, K. Vogtmann. Abelian covers of graphs and maps between outer automorphism groups of free groups. Mathematische Annalen 353 (2012), no. 4, 1069-1102.

[6] E. H. Brown, R. H. Szczarba. On the rational homotopy type of function spaces. Trans. Amer. Math. Soc., 349 (1997), 4931-4951.

[7] U. Buijs, Y. Félix, A. Murillo. $L_{\infty}$ models of based mapping spaces. J. Math. Soc. Japan 63 (2011), no. 2, 503-524.

[8] G. Carlsson, C. Douglas, B. I. Dundas. Higher topological cyclic homology and the Segal conjecture for tori. Adv. Math. 226 (2011), no. 2, 1823-1874.

[9] Y. Félix, S. Halperin, J.-C. Thomas. Rational homotopy theory. Graduate Texts in Mathematics, 205. Springer-Verlag, New York, 2001. xxxiv +535 pp.

[10] Y. Félix, S. Halperin, J.-C. Thomas. Rational homotopy theory II. World Scientific Publishing Co. Pte. Ltd., Hackensack, NJ, 2015. xxxvi+412 pp.

[11] B. Fresse. Modules over operads and functors. Lecture Notes in Mathematics, 1967. Springer-Verlag, Berlin, 2009. x+308 pp.

[12] S. M. Gersten. A presentation for the special automorphism group of a free group. J. Pure Appl. Algebra, 33(3):269-279, 1984.

[13] M. Gerstenhaber, S. D. Schack. A Hodge-type decomposition for commutative algebra cohomology. J. Pure Appl. Algebra 48 (1987), no. 3, 229-247.

[14] G. Ginot. Notes on factorization algebras, factorization homology and applications. Mathematical aspects of quantum field theories, 429-552, Math. Phys. Stud., Springer, Cham, 2015..

[15] G. Ginot, T. Tradler, M. Zeinalian. A Chen model for mapping spaces and the surface product. Ann. Sci. Ec. Norm. Supér. (4) 43 (2010), no. 5, 811-881.

[16] G. Ginot, T. Tradler, M. Zeinalian. Higher Hochschild homology, topological chiral homology and factorization algebras. Comm. Math. Phys. 326 (2014), no. 3, 635-686.

[17] F. Grunewald, A. Lubotzky. Linear representations of the automorphism group of a free group. Geom. Funct. Anal. 18(5):1564-1608, 2009.

[18] M. Intermont, B. Johnson, R. McCarthy. The rank filtration and Robinson's complex. J. Pure Appl. Algebra 212 (2008), no. 4, 735-752.

[19] D. Kielak. Outer automorphism groups of free groups: linear and free representations. J. Lond. Math. Soc. (2) 87(3):917-942, 2013.

[20] D. Kielak. Low dimensional free and linear representations of $\operatorname{Out}\left(F_{3}\right)$. J. Group Theory 18 (2015), no. 6, 913-949.

[21] J.-L. Loday. Opérations sur l'homologie cyclique des algèbres commutatives. Invent. Math. 96 (1989), no. 1, 205-230.

[22] J.-L. Loday and B. Vallette. Algebraic Operads. Number 346 in Grundlehren der mathematischen Wissenschaften. Springer, Heidelberg, 2012.

[23] J. Lurie. On the classification of topological field theories. Current developments in mathematics, 2008, 129-280, Int. Press, Somerville, MA, 2009.

[24] J. McCool. A presentation for the automorphism group of a free group of finite rank. J. London Math. Soc. (2), 8:259-266, 1974.

[25] F. Patras. Generic algebras and iterated Hochschild homology. J. Pure Appl. Algebra 162 (2001), no. 2-3, 337-357.

[26] F. Patras, J.-C. Thomas. Cochain algebras of mapping spaces and finite group actions. Topology Appl. 128 (2003), no. 2-3, $189-207$.

[27] T. Pirashvili, Dold-Kan type theorem for Г-groups, Math. Ann. 318 (2000), no. 2, 277-298.

[28] T. Pirashvili, Hodge decomposition for higher order Hochschild homology, Ann. Sci. Ecole Norm. Sup (4) 33 (2000), no. 2, 151-179.

[29] C. Schlichtkrull. Higher topological Hochschild homology of Thom spectra. J. Topol. 4 (2011), no. 1, 161-189.

[30] P.-A. Songhafouo Tsopméné. The rational homology of spaces of long links. Preprint arXiv:1312.7280

[31] P.-A. Songhafouo Tsopméné, V. Turchin. Hodge decomposition in the rational homology and homotopy of high dimensional string links. Preprint arXiv:1504.00896

[32] V. Turchin. Hodge-type decomposition in the homology of long knots. J. Topol., 3(3):487-534, 2010.

[33] V. Turchin, T. Willwacher. Relative (non-)formality of the little cubes operads and the algebraic Cerf lemma, arXiv:1409.0163, 2014.

[34] V. Turchin, T. Willwacher. Commutative hairy graphs and representations of $\operatorname{Out}\left(F_{r}\right)$, arXiv:1603.08855 2016.

[35] M. Weiss. Embeddings from the point of view of immersion theory. I. Geom. Topol. 3 (1999), 67-101.

Department of Mathematics, Kansas State University, 138 Cardwell Hall, Manhatan, KS 66506, USA

E-mail address: turchin@ksu.edu

Institute of Mathematics, University of Zurich, Winterthurerstrasse 190, 8057 Zurich, Switzerland

E-mail address: thomas.willwacher@math.uzh.ch 